\title{
Numerical Simulations of Tropical Cyclone-Ocean Interaction With a High-Resolution Coupled Model
}

\author{
Morris A. Bender, ${ }^{1}$ IsAaC Ginis, ${ }^{2,3}$ AND Yoshio Kurihara ${ }^{1}$
}

\begin{abstract}
The tropical cyclone-ocean interaction was investigated using a high-resolution tropical cyclone ocean coupled model. The model design consisted of the NOAA Geophysical Fluid Dynamics Laboratory tropical cyclone prediction model which was coupled with a multilayer primitive equation ocean model. Coupling between the hurricane and the ocean models was carried out by passing into the ocean model the wind stress, heat, and moisture fluxes computed in the hurricane model. The new sea surface temperature (SST) calculated by the ocean model was then used in the tropical cyclone model. A set of idealized numerical experiments were performed in which a tropical cyclone vortex was embedded in both easterly and westerly basic flows of $2.5,5$, and $7.5 \mathrm{~m} \mathrm{~s}^{-1}$ with a fourth experiment run with no basic flow specified initially. The profile of the tangential wind for Hurricane Gloria at 1200 UTC 22, September 1985 was used as the initial condition of the tropical cyclone for each of the experiments. The model ocean was initially horizontally homogenous and quiescent. To clarify the impact of the ocean response to the hurricane's behavior, analogous experiments were also carried out with the SST kept constant (control cases). The experiments indicated that the cooling of the sea surface induced by the tropical cyclone resulted in a significant impact on the ultimate storm intensity due to the reduction of total heat flux directed into the tropical cyclone above the regions of decreased SST. The sea surface cooling produced by the tropical cyclones was found to be larger when the storms moved slower. In the experiments run without an initial basic flow, the maximum SST anomaly was about $-5.6^{\circ} \mathrm{C}$ with a resulting difference in the minimum sea level pressure and maximum surface winds of $16.4 \mathrm{hPa}$ and $-7 \mathrm{~m} \mathrm{~s}^{-1}$, respectively. In contrast, in the experiments run with the 7.5 $\mathrm{m} \mathrm{s}^{-1}$ basic flow, the maximum SST anomalies ranged from about $2.6^{\circ}$ to $3.0^{\circ} \mathrm{C}$ with a difference in the minimum sea level pressure and maximum surface winds of about $7.3 \mathrm{hPa}$ and $-2.7 \mathrm{~m} \mathrm{~s}^{-1}$. The tropical cyclone-ocean coupling significantly influenced the storm track only for the case with no basic flow and the $2.5 \mathrm{~m} \mathrm{~s}^{-1}$ easterly flow. In these cases the storm with the ocean interaction turned more to the north and east (no basic flow) or the north $\left(2.5 \mathrm{~m} \mathrm{~s}^{-1}\right.$ easterly flow) of the experiments with constant SST. In the first case, the storm by 72 hours was located over $70 \mathrm{~km}$ to the east-southeast of the control case. A possible explanation for this track deviation is related to a systematic weakening of the mean tangential flow at all radii of the storm due to the interaction with the ocean and resulting alteration of the beta drift.
\end{abstract}

\section{INTRODUCTION}

The importance of the sea surface temperature (SST) in the genesis and intensity of tropical cyclones has become well established. It is known that tropical cyclones usually develop over waters in which the SST is $26^{\circ} \mathrm{C}$ or higher. Both theoretical [e.g., Emanuel, 1986] and numerical models [e.g., Chang, 1979; Tuleya and Kurihara, 1982] confirm the sensitivity of storm intensity to the SST in the vicinity of storms.

The tropical cyclone-ocean system is one with positive and negative feedbacks. During the genesis and development stages a positive feedback in the tropical cyclone-ocean system exists. As the tropical cyclone strengthens, the evaporation rate grows due to the increase in the surface wind speed. The enhancement of the moisture supply from the ocean leads to an increase of the latent heat energy that drives the circulation of the tropical cyclone. However, as the storm continues to intensify, the increasing surface wind stress generates strong turbulent mixing and deepening of

\footnotetext{
${ }^{1}$ NOAA Geophysical Fluid Dynamics Laboratory, Princeton University, Princeton, New Jersey.

${ }^{2}$ Program in Atmospheric and Oceanic Sciences, Princeton University, Princeton, New Jersey.

${ }^{3}$ Now at Graduate School of Oceanography, University of Rhode Island, Narragansett, Rhode Island.
}

Copyright 1993 by the American Geophysical Union.

Paper number 93JD02370.

0148-0227/93/93JD-02370\$05.00 the ocean mixed layer. This causes significant decreases in the SST due to entrainment of the cooler waters from the thermocline into the mixed layer. The observed SST anomalies induced by the tropical cyclone have been observed to vary from $1^{\circ} \mathrm{C}$ to $6^{\circ} \mathrm{C}$ [e.g., Black, 1983]. The cooling of the sea surface results in reduction in the total heat flux (latent plus sensible) into the atmosphere leading to a decrease in storm intensity. This process represents a negative feedback mechanism.

A number of numerical simulation studies have been made in order to understand and to evaluate various aspects of the ocean response to moving tropical cyclones [e.g., Chang and Anthes, 1978; Price, 1981; Ginis and Dikinov, 1989; Cooper and Thompson, 1989]. In the works of Price [1981] and Ginis and Dikinov [1989] a realistic ocean response to Hurricane Eloise (1975) and Typhoon Virginia (1978) was simulated, respectively.

However, numerical simulations of tropical cyclone-ocean interaction have been rather limited. In earlier coupled experiments performed by Chang and Anthes [1979] and Sutyrin and Khain [1979], an axisymmetric tropical cyclone model was coupled with an upper mixed layer ocean model. Results indicated that over a short period of time the sea surface cooling did not have a large effect on the tropical cyclone's dynamics. For example during a 1-day period of the integrations carried out by Chang and Anthes [1979], the minimum sea level pressure rose only by $2 \mathrm{hPa}$. Sutyrin and Khain [1984] performed a series of numerical experiments with different speeds of tropical cyclone motion and reported that the model storms responded with about a 1-day delay to 
TABLE 1. Grid System of the Triply Nested Mesh Hurricane Model

\begin{tabular}{|c|c|c|c|c|c|c|}
\hline \multirow[b]{3}{*}{ Mesh } & \multirow{3}{*}{$\begin{array}{c}\text { Grid } \\
\text { Resolution, } \\
\text { deg }\end{array}$} & \multicolumn{4}{|c|}{ Domain Size } & \multirow{3}{*}{$\begin{array}{l}\text { Time } \\
\text { Step, }\end{array}$} \\
\hline & & \multicolumn{2}{|c|}{ Longitude } & \multicolumn{2}{|c|}{ Latitude } & \\
\hline & & Deg & Points & Deg & Points & \\
\hline 1 & 1 & 55 & (55) & 55 & (55) & 120 \\
\hline 2 & $1 / 3$ & 15 & (45) & 15 & (45) & 40 \\
\hline 3 & $1 / 6$ & $81 / 3$ & (50) & $81 / 3$ & (50) & 20 \\
\hline
\end{tabular}

the decrease of the SST, although the weakening of the storms was larger (i.e., 5-10 hPa), especially for slowly moving storms. In all of these previous studies the tropical cyclones were represented by axisymmetric models with rather coarse resolution.

Ginis et al. [1989] developed a three-dimensional coupled model by coupling a five-level tropical cyclone model [Khain, 1988] and a three-layer primitive equation ocean model [Ginis and Dikinov, 1989]. These integrations were carried out without a basic environmental flow. The storm motion was due only to the beta effect. It was shown that the cooling of the sea surface under the tropical cyclone led not only to a decrease in its intensity but also to a change in the speed of the storm motion. According to Khain and Ginis [1991] the effect of a basic flow was crudely taken into account by moving the atmosphere grid over the ocean grid with prescribed velocities. Both the intensity (i.e., 5-7 hPa) and the motion of the tropical cyclone were affected by the cooling of the sea surface (i.e., $3.5^{\circ}-6^{\circ} \mathrm{C}$ ) induced by the tropical cyclone. They found that the model storm with fixed SST tended to be displaced to the right, whereas those in the coupled model tended to be displaced to the left of the direction of the atmospheric grid motion. They concluded that such a difference was apparently due to an asymmetry of the energy supply from the ocean.

Although previous studies based on these simple coupled tropical cyclone-ocean models indicated that the negative feedback between the ocean and the tropical cyclone may indeed be substantial, the details of this interaction have not been studied thoroughly. More thorough numerical investigation of this problem required the use of a very high resolution atmosphere-ocean model. Such a model has been developed at the NOAA Geophysical Fluid Dynamics Laboratory.

The main goal of the present study is to explore the effect of the tropical cyclone-ocean interaction on the surface heat flux, intensity, and track of tropical cyclones based on numerical results from the GFDL high-resolution tropical cyclone-ocean coupled model. Analysis of the results will also include the dynamical response of the ocean to the tropical cyclone.

A brief description of the tropical cyclone and ocean models will be presented in section 2 . Verification of the ocean model with observations will also be presented in this section. The initial conditions of the tropical cyclone and ocean models, experimental design, and classification of experiments will be discussed in section 3 . The numerical results of the ocean response will be presented in section 4 with the results from the tropical cyclone-ocean coupled model detailed in section 5. A summary of the results and concluding remarks will be reported in section 6 .

\section{Model Description}

\subsection{Tropical Cyclone Model Description}

The multiply nested movable mesh (MMM) model described by Kurihara and Bender [1980] was used for all of the time integrations of the tropical cyclone model. Specific model details have been outlined in previous publications [e.g., Tuleya et al., 1984; Bender et al., 1987]. The model is a primitive equation model formulated in latitude, longitude, and $\sigma$ coordinates, with 18 levels in the vertical [i.e., Kurihara et al., 1990, Table 1]. The grid system for each of the triply nested meshes in the present study is summarized in Table 1. The outermost domain ranged from the equator to $55^{\circ} \mathrm{N}$ in the meridional direction. The model physics include cumulus parameterization described by Kurihara [1973] with some additional modifications [Kurihara and Bender, 1980, Appendix C], a Monin-Obukhov scheme for the surface flux calculation, and the Mellor and Yamada [1974] level-2 turbulence closure scheme for the vertical diffusion, with a background diffusion coefficient added. Some of the recent modifications to the model that were reported in section 2.1 of Bender et al. [1993] were added as well. In particular, the Charnock constant which is used in the calculation of the roughness length has been changed to an updated value of $.0185[W u, 1982]$. The three-point smoothing operator used to suppress numerical noise was modified to include a desmoothing operator. The effect of radiative transfer was not treated explicitly. However, the zonal mean temperature was adjusted toward its initial value using a Newtonian-type damping with a 24-hour damping period. The simulation capability of this model has been well demonstrated in experiments using real data [e.g., Kurihara et al., 1990].

\subsection{Ocean Model Description}

The ocean model is a primitive equation multilayer stratified model formulated in the spherical coordinate system. The model was developed from a three-layer reduced gravity model that is described by Ginis and Dikinov [1989] and currently includes a free surface and flat bottom. The vertical ocean structure is represented by a surface mixed layer and a specified number of layers below (seven for the current set of experiments). The mixed layer is considered as a turbulent boundary layer which exchanges momentum and heat with the atmosphere at its surface and with the thermocline by entrainment at its base. Normally, the mixed layer is well mixed due to turbulent mixing and is vertically homogeneous in temperature. It is assumed that below the mixed layer, in the thermocline and deep water, the temperature is linearly depth dependent within each layer. The interfacial (transition) layer with a sharp change of temperature at the base of the mixed layer is modeled by a discontinuity surface 
(a velocity and density jump across the mixed layer base). The vertical turbulent mixing at the mixed layer base is computed from the scheme formulated by Deardorff [1983]. The horizontal velocity vector is assumed to be independent of depth within each layer. Since the present numerical experiments were designed to simulate the effects of tropical cyclone-ocean interaction in the deep open ocean, the effects of bottom topography and coastal geography were not considered. A detailed description of the ocean model and the numerical methods used for solving the governing equations are given in the Appendix.

The ocean model used a mesh of constant grid spacing of $1 / 6^{\circ}$ which was equal to the resolution of the finest grid of the hurricane model. The sizes of the ocean computational domain varied from one experiment to another depending on the translational speed of the storm. On the average it spanned about $35^{\circ}$ in the longitudinal direction and about $21^{\circ}$ in the latitudinal direction. The time step of the ocean model for all the experiments was set to $10 \mathrm{~min}$.

\subsection{Ocean Model Verification}

The ocean model performance was first tested without tropical cyclone interaction, using the field observations of the ocean response to Hurricane Norbert (1984), described in detail by Sanford et al. [1987]. The data set is unique because, for the first time, simultaneous measurements of atmospheric and oceanic parameters were carried out in the region of direct hurricane forcing. The model integration began from 0000 UTC, September 22, 1984, when the storm was located at $16.5^{\circ} \mathrm{N}, 111.9^{\circ} \mathrm{W}$ and ended at $0000 \mathrm{UTC}$, September 24,1984 , when the survey took place. The storm was assumed to move along the Norbert best track without changing its structure and strength. The translational speed of the storm was determined from the storm position each 6 hours and is shown in Figure 1 $a$. The axisymmetric surface wind stress profile was obtained based on research flightlevel $(1500 \mathrm{~m})$ winds provided by P. G. Black (personal correspondence), Hurricane Research Division/NOAA. The computational domain included $100 \times 100$ grid points with $1 / 6^{\circ}$ grid spacing. Since the Norbert survey region was not dynamically very active, we assumed that the ocean was initially homogeneous and quiescent. The SST and the mixed layer depth were initially set to $28.5^{\circ} \mathrm{C}$ and $30 \mathrm{~m}$, respectively, and the temperature jump across the bottom of the mixed layer was set to $1^{\circ} \mathrm{C}$. The temperature profile specified in the thermocline was typical for the eastern Pacific. The initial layer thicknesses below the mixed layer were set to $45,50,70,100,150$, and $250 \mathrm{~m}$. In Figures $1 b$ and $1 c$ the simulated SST deviations and the mixed layer current field are compared with observations. The largest observed mixed layer velocities were $1.1 \mathrm{~m} \mathrm{~s}^{-1}$, while numerical calculations gave a value of $1.06 \mathrm{~m} \mathrm{~s}^{-1}$. The model sea surface cooling was also very close to the observed values although the simulation indicates that the largest SST decrease occurred outside of the survey region where the storm made a sharp turn toward the north. The simulated and observed ocean response demonstrated a significant rightward bias in the current speed, temperature deviation, and mixed layer depth fields relative to the storm track. This is in accord with other hurricane observations and models [e.g., Black, 1983; Chang and Anthes, 1978; Price, 1981]. The asymmetry in the velocity pattern is a result of effective
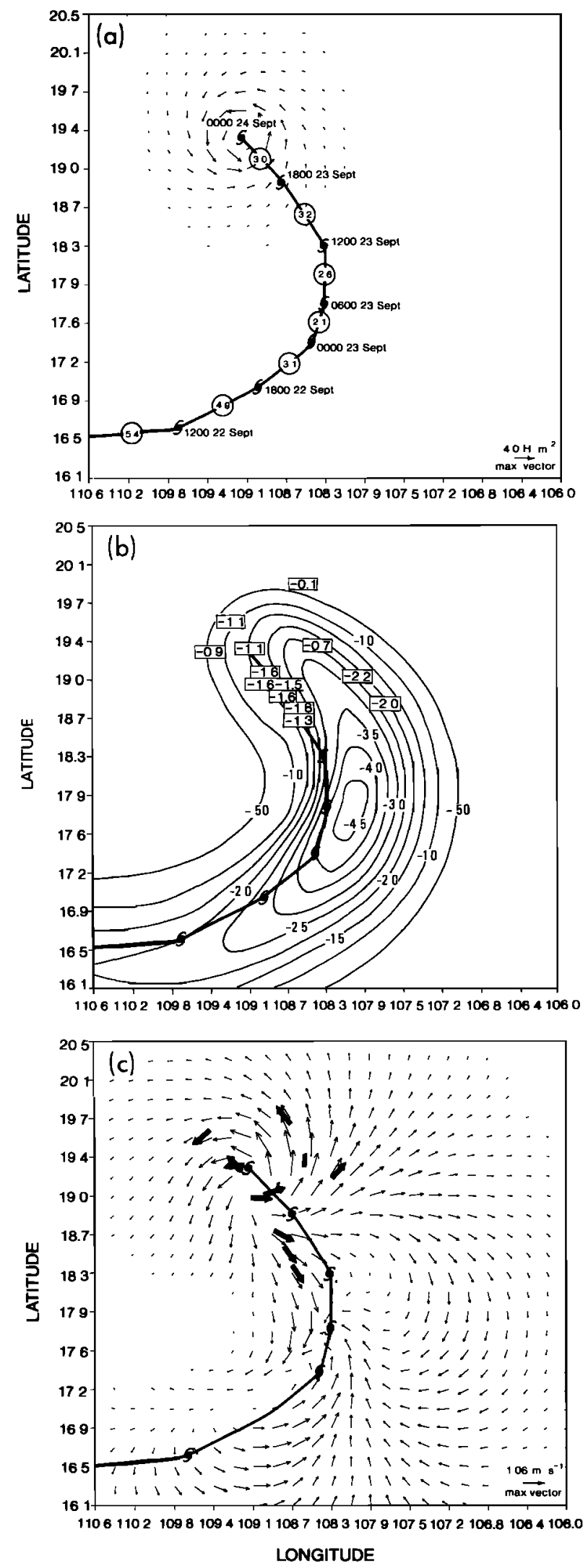

Fig. 1. Ocean model response to Hurricane Norbert valid 0000 UTC, September 24, 1984. (a) The observed storm track and the vectors of the wind stress. The estimated speed of the storm motion $\left(\mathrm{m} \mathrm{s}^{-1}\right)$ is shown inside the circles. $(b)$ The model-computed sea surface temperature (SST) anomalies $\left({ }^{\circ} \mathrm{C}\right.$ ) with observed values inside the rectangles. (c) Vectors of the mixed layer velocities. The solid arrows indicate the observed velocities. 
coupling of clockwise rotating wind stress vectors on the right side of the track and inertially rotating wind-driven currents. This dynamic field asymmetry introduces asymmetry in the turbulent mixing (entrainment) which is mainly responsible for sea surface cooling. The overall comparison of the model simulations with observations suggests that the present ocean model is capable of reproducing the main features of the ocean response to the tropical cyclone forcing, particularly the observed ocean currents and sea surface cooling.

\section{Initial Condition and Experimental Design}

\subsection{Tropical Cyclone Model Initial Condition}

The experiments were designed in order that a tropical cyclone could move over the ocean in prescribed easterly and westerly zonal atmospheric flows. The zonal flows had constant angular velocities which corresponded to zonal speeds of $7.5,5.0$, and $2.5 \mathrm{~m} \mathrm{~s}^{-1}$ at $18^{\circ} \mathrm{N}$ (initial latitude of the tropical cyclone center for each experiment). The zonal flows (identified as the basic flows) were constant with height and extended throughout the entire depth of the atmosphere. The environmental condition surrounding Hurricane Gloria on 1200 UTC, September 22, 1985, served as the initial environmental condition of the mass and moisture fields for all of these experiments. Specifically, the basic field of temperature, relative humidity at each level in the vertical, and sea level pressure were computed from the NMC T80 global analysis for a region of $5^{\circ}$ square surrounding the observed center position of Hurricane Gloria. The resulting initial environmental sounding is presented in Figure $2 a$.

The symmetric vortex was generated from time integration of an axisymmetric version of the hurricane model, following the method outlined in section 4a of Kurihara et al. [1993] and using the tangential wind profile for Gloria on 1200 UTC, September 22, 1985, as input. In their scheme the tangential wind field is forced during the integration toward the observed storm tangential wind profile while the moisture, mass, and radial wind profiles are free to develop a model consistent structure. The obtained symmetric vortex was then placed onto the basic fields at $18^{\circ} \mathrm{N}$. After the symmetric vortex was added to the basic flow, the mass field (temperature and sea level pressure) was recomputed through use of the static initialization method outlined in section 5 of the above paper.

\subsection{Ocean Model Initial Condition}

In all of the experiments the ocean was initially assumed to be horizontally homogeneous and quiescent. Hence the initial velocity fields were set to zero within all model layers. The observed data collected by Black [1983] was used to specify the vertical thermal structure. A wide range of stratifications occur within the different ocean basins. However, there is a similarity for most of the observed cases in the western North Atlantic, Caribbean, Saragasso Sea, Gulf of Mexico, and the eastern North Pacific. In particular, the majority of temperature profiles in these regions before passage of tropical cyclones are characterized by a shallow (near $40 \mathrm{~m}$ ) and warm (about $28^{\circ} \mathrm{C}$ ) mixed layer and steep temperature gradients in the seasonal thermocline. Thus a particular prestorm profile might be representative of the typical ocean structure before a tropical cyclone passage.
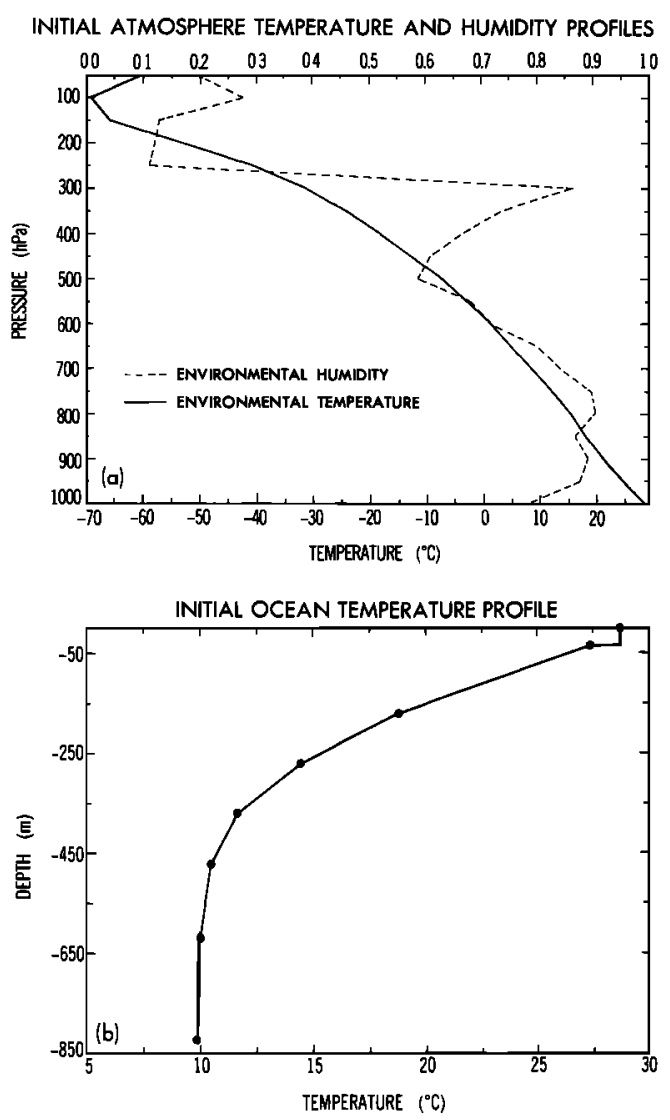

Fig. 2. (a) The initial vertical profile of the environmental relative humidity and temperature $\left({ }^{\circ} \mathrm{C}\right)$ used in the atmosphere model and $(b)$ the initial vertical profile of the temperature $\left({ }^{\circ} \mathrm{C}\right)$ used in the ocean model for all the experiments presented in this study. The initial depth of each layer interface in the ocean model is indicated by circles.

Accordingly, an initial temperature profile was constructed for all of our idealized experiments (Figure $2 b$ ) which was typical for tropical regions of the western North Atlantic. The SST was set to $28.85^{\circ} \mathrm{C}$ (the approximate average SST observed before the passage of Hurricane Gloria (1985) during its deepening stage); the mixed layer thickness was set to $40 \mathrm{~m}$ with a temperature jump of $1.0^{\circ} \mathrm{C}$ across the base of the mixed layer. The initial thicknesses of each layer below the mixed layer were set to $140,100,100,100,150$, and $220 \mathrm{~m}$.

\subsection{Experimental Design and Summary of Experiments}

Both the outer domain of the tropical cyclone model and the entire domain of the ocean model were fixed throughout the integrations, while the inner meshes of the tropical cyclone model moved with the storm center.

The method of coupling between the tropical cyclone and the ocean models was performed as follows: During the period of one ocean model time step, the tropical cyclone model was integrated keeping the SST constant. The wind stress, heat, and moisture fluxes computed in the tropical cyclone model were passed into the ocean model. The ocean model was then integrated one step and a new SST was calculated. The new SST was then used in the ensuing time steps of the tropical cyclone model. The transfer of the wind 
stress, heat, and moisture fluxes from the tropical cyclone model to the fixed grid system of the ocean model as well as the transfer of the SST field from the ocean to the tropical cyclone model were accomplished through bilinear interpolation.

The interaction of the tropical cyclone with the ocean was included from the beginning of the integrations in the primary experiments. In addition, two supplementary experiments were carried out as well. In the first supplementary experiment no basic flow was included. Thus the storm was motionless at the beginning of the integration and the storm motion developed as the beta gyre (the dipolar vorticity caused by the advection of planetary vorticity) evolved. In the second supplemental experiment the interaction with the ocean was introduced after integration of the tropical cyclone model with constant SST for 24 hours. Finally, to clarify the impact of the ocean response onto the storm's behavior, analogous experiments were carried out with the SST kept constant (control experiments without coupling). A summary of all the experiments is included in Table 2 . All of the integrations presented in this study were extended to 72 hours.

\section{Ocean Response in the Tropical Cyclone-Ocean COUPLED MODEL}

The primary issue in the present study is the feedback effect on the tropical cyclone produced by the cooling of the SST. In general, the changes in the SST are strongly dependent on the currents in the upper part of the ocean. Therefore the discussion will begin with essential features of the dynamical response of the ocean to the tropical cyclones.

The model currents in the mixed layer at 72 hours are presented in Figure $3 a$ for the experiment with $5 \mathrm{~m} \mathrm{~s}^{-1}$ easterly basic flow. In the front of the tropical cyclone the cyclonically rotating wind stress generated mixed layer currents which were oriented in nearly the same direction as the wind stress. However, the current pattern became significantly asymmetric behind the tropical cyclone. This asymmetry, first identified by Chang and Anthes [1978] occurred because the clockwise inertially rotating flow was effectively accelerated on the right side and decelerated on

TABLE 2. Summary of the Numerical Experiments

\begin{tabular}{lcl}
\hline $\begin{array}{c}\text { Direction of } \\
\text { Basic Flow }\end{array}$ & $\begin{array}{c}\text { Magnitude of } \\
\text { Basic Flow, } \\
\text { m s }\end{array}$ & \multicolumn{1}{c}{$\begin{array}{c}\text { Experiment } \\
\text { Description }\end{array}$} \\
\hline Easterly & 2.5 & coupled \\
Easterly & 2.5 & noncoupled \\
Easterly & 5.0 & coupled \\
Easterly & 5.0 & noncoupled \\
Easterly & 7.5 & coupled \\
Easterly & 7.5 & noncoupled \\
Westerly & 2.5 & coupled \\
Westerly & 2.5 & noncoupled \\
Westerly & 5.0 & coupled \\
Westerly & 5.0 & noncoupled \\
Westerly & 7.5 & coupled \\
Westerly & 7.5 & noncoupled \\
& & Supplemental Experiments \\
No basic flow & none & coupled \\
No basic flow & none & noncoupled \\
Easterly & 5.0 & coupled after 24 hours \\
\hline
\end{tabular}
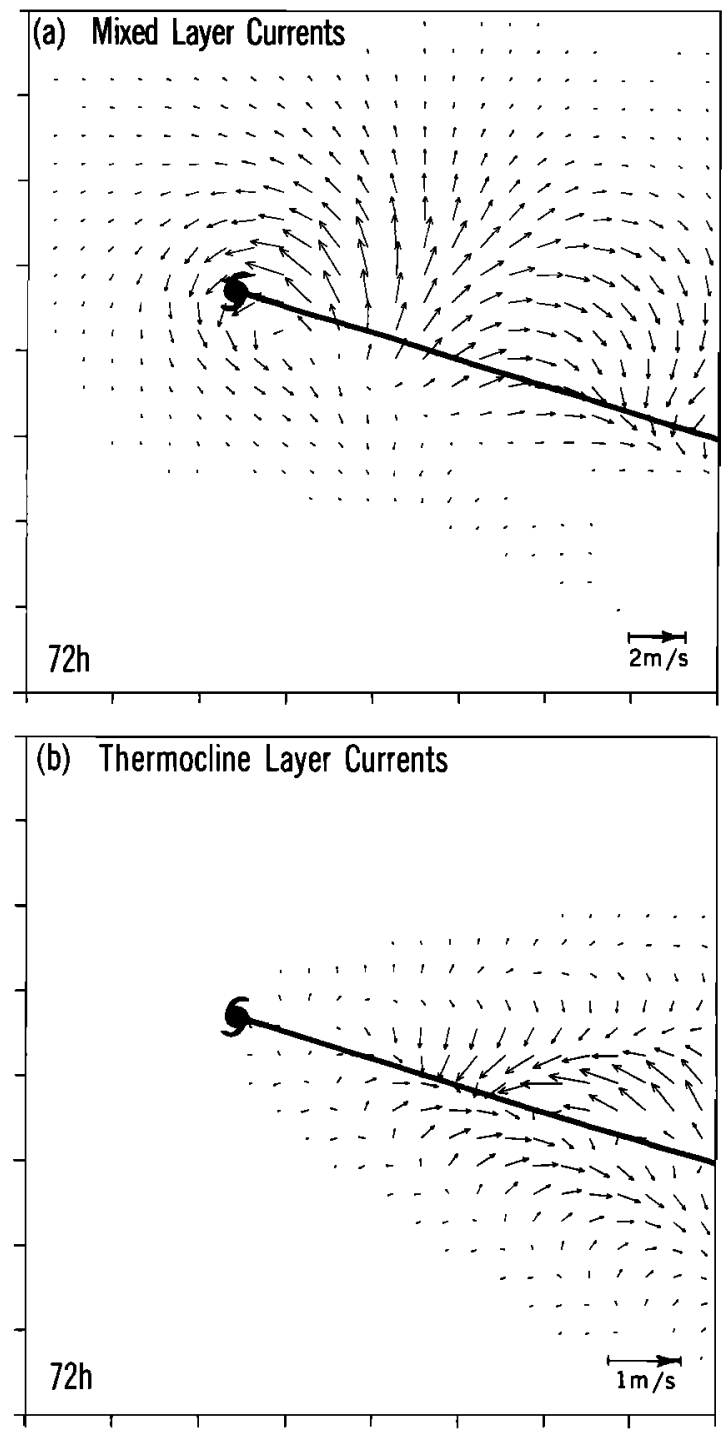

Fig. 3. The current velocities in $(a)$ the mixed layer and $(b)$ the uppermost thermocline layer at 72 hours for the integration with $5 \mathrm{~m}$ $\mathrm{s}^{-1}$ easterly basic flow. The region shown is for a portion of the ocean domain with the tick marks drawn at $1^{\circ}$ intervals. The hurricane center position at 72 hours is indicated by the hurricane symbol with the storm track indicated by the solid line.

the left side due to the rotation of the wind stress vectors produced by the moving tropical cyclone. The resulting mixed layer flow makes an important contribution to the horizontal transport process. In addition, it can induce turbulent mixing at the mixed layer base through the shear instability, causing the entrainment of thermocline water. This process, as discussed below, is very important in the changes of the temperature and the thickness of the mixed layer. Another important feature of the mixed layer flow is that it tends to be divergent when the speed of the inertiagravity waves $\left(1-2 \mathrm{~m} \mathrm{~s}^{-1}\right)$ is smaller than the hurricane translational speed [Geisler, 1970]. The mixed layer divergence generated vertical motion that extended downward into the thermocline (Figure $4 a$ ).

The vertical motion induced by the tropical cyclone consists of two distinct components: the steady upwelling that resulted from the Ekman pumping forced by the wind stress 

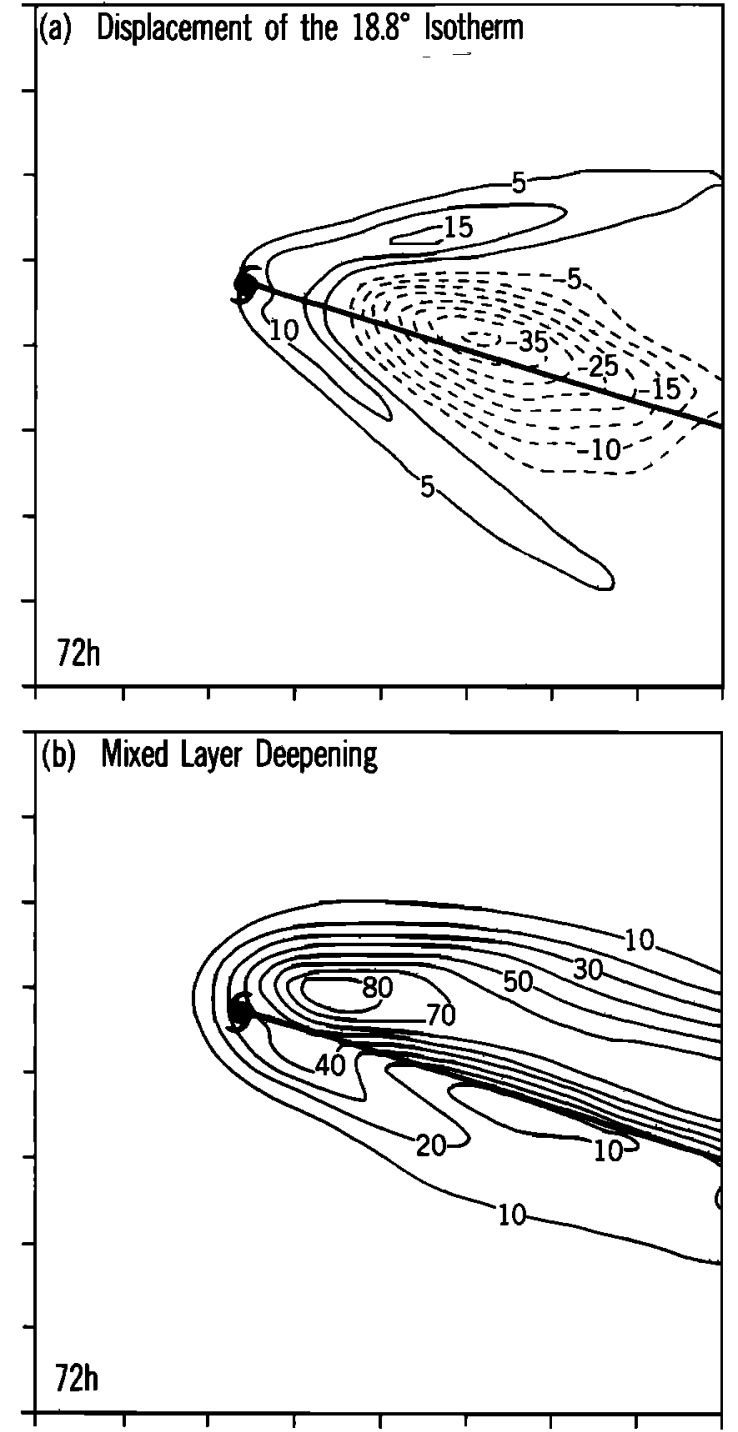

Fig. 4. (a) The 72-hour displacement (meters) of the $18.8^{\circ} \mathrm{C}$ isotherm (located at the bottom of the second ocean model layer) from its initial position (dashed contours correspond to upward motion) and (b) the 72-hour mixed layer deepening (meters) for the coupled experiment with the $5 \mathrm{~m} \mathrm{~s}^{-1}$ easterly basic flow. The region shown is for a portion of the ocean domain with the tick marks drawn at $1^{\circ}$ intervals. The hurricane center position at 72 hours is indicated by the hurricane symbol with the storm track indicated by the solid line.

components parallel to the storm track and near-inertial frequency oscillations characterized by a pattern of cells of upwelling and downwelling behind the storm. In Figure $4 a$, only the upwelling phase of these cells is observed with maximum displacement of the $18.8^{\circ}$ isotherm located about $350 \mathrm{~km}$ behind the storm center. The arrowhead shape of the band of compensating downwelling indicates that horizontal dispersion of inertia-gravity waves was taking place after the storm passage.

The thermocline current pattern at 72 hours (Figure $3 b$ ) showed an entirely different pattern from the mixed layer currents, indicating that the dynamics of the two currents were considerably different. This fact is important in determining the shear instability across the mixed layer base, the primary source of turbulent mixing which leads to the SST decrease. While the mixed layer currents were a result of wind stress effects, the thermocline currents were gradient currents induced by the pressure gradients produced from the mixed layer divergence. In the present deep ocean simulations, the currents in the thermocline primarily developed far from the tropical cyclone center. This feature is in accord with recent observations taken after the passage of Hurricane Gay [Church et al., 1989] and a model study by Price [1983].

The strong mixed layer depth deepening underneath the storm (Figure $4 b$ ) was a consequence of both the vertical advection (downwelling) and the entrainment of the thermocline water. The maximum deepening, located about $180 \mathrm{~km}$ to the east of the storm center, slightly exceeded $80 \mathrm{~m}$. This is somewhat larger than reported in other previous numerical [e.g., Price, 1981] and observational studies [e.g., Sanford et al., 1987] of the ocean response to tropical cyclones. However, the tropical cyclone in this experiment was quite intense with maximum wind stresses ranging from 5.5 to 5.8 $\mathrm{N} \mathrm{m}^{-2}$ during the integration period. The rightward bias of the mixed layer deepening is in good agreement with previous numerical studies and is well correlated with the rightward bias in the mixed layer currents. This suggests that current shear instability is the principal mechanism producing the mixed layer turbulence after the initial stage of the tropical cyclone forcing. Well behind the storm the mixed layer deepening is substantially reduced due to weakening of the turbulent mixing and the intensifying of the upwelling.

The SST anomalies produced by the tropical cyclone are shown at 72 hours (Figure 5) for two of the numerical experiments. In both cases a pronounced rightward bias exists in the sea surface cooling with respect to the storm track. This asymmetry is correlated with the asymmetry in the mixed layer currents (Figure $3 a$ ) and mixed layer deepening (Figure $4 b$ ), and again suggests that the entrainment was the major cause of the SST cooling. Indeed, Figure 6 shows that in the present model the maximum heat flux due to the entrainment at the mixed layer base at 72 hours was about 30 times larger than the maximum total surface heat fluxes (latent plus sensible). However, it should be noted that the surface heat fluxes could make a substantial contribution to turbulent mixing through convective mechanisms. This mechanism might become important in the front and left quadrants of the storm where the wind-driven currents are relatively weak.

In both cases in Figure 5 the region of maximum SST anomalies was located well behind the storm center. Directly underneath the storm center the SST decrease varied from about $1.5^{\circ}$ to about $3^{\circ} \mathrm{C}$ for the two experiments. This location of the maximum sea surface cooling was mainly due to the fact that the turbulent mixing and entrainment continued well after passage of the storm since the shear instability of the inertial currents was maintained and the shear could be enhanced by development of gradient currents below the mixed layer. The position of maximum SST decrease was different from that of the maximum mixed layer deepening because the vertical advection had little influence on the SST change.

Finally, it is important to mention that the SST response was quite sensitive to the basic flow speed. With no basic flow the sea surface cooling (Figure 5 , bottom) was much stronger and more extensive than the experiment with the 5 $\mathrm{m} \mathrm{s}^{-1}$ easterly basic flow (Figure 5 , top). The extension of 
the area of cooling due to the slower-moving tropical cyclone was a result of the increased amount of time that the wind stress was able to act on a fixed ocean point. This lead to intensification of the drift currents and, as a consequence, to an increase of the velocity shear at the base of the mixed layer. Therefore even a relatively weak surface wind at the storm periphery could generate turbulent mixing sufficient for entrainment, resulting in a larger area with decreased SST. The magnitude of the SST response for the different basic flows will be addressed in greater detail in section 5 .

\section{Tropical Cyclone Response to Air-Sea Interaction}

In this section the effect of the ocean coupling on the surface heat flux, storm intensity, and motion will be evalu-

\section{SST ANOMALIES $\left({ }^{\circ} \mathrm{C}\right)$}
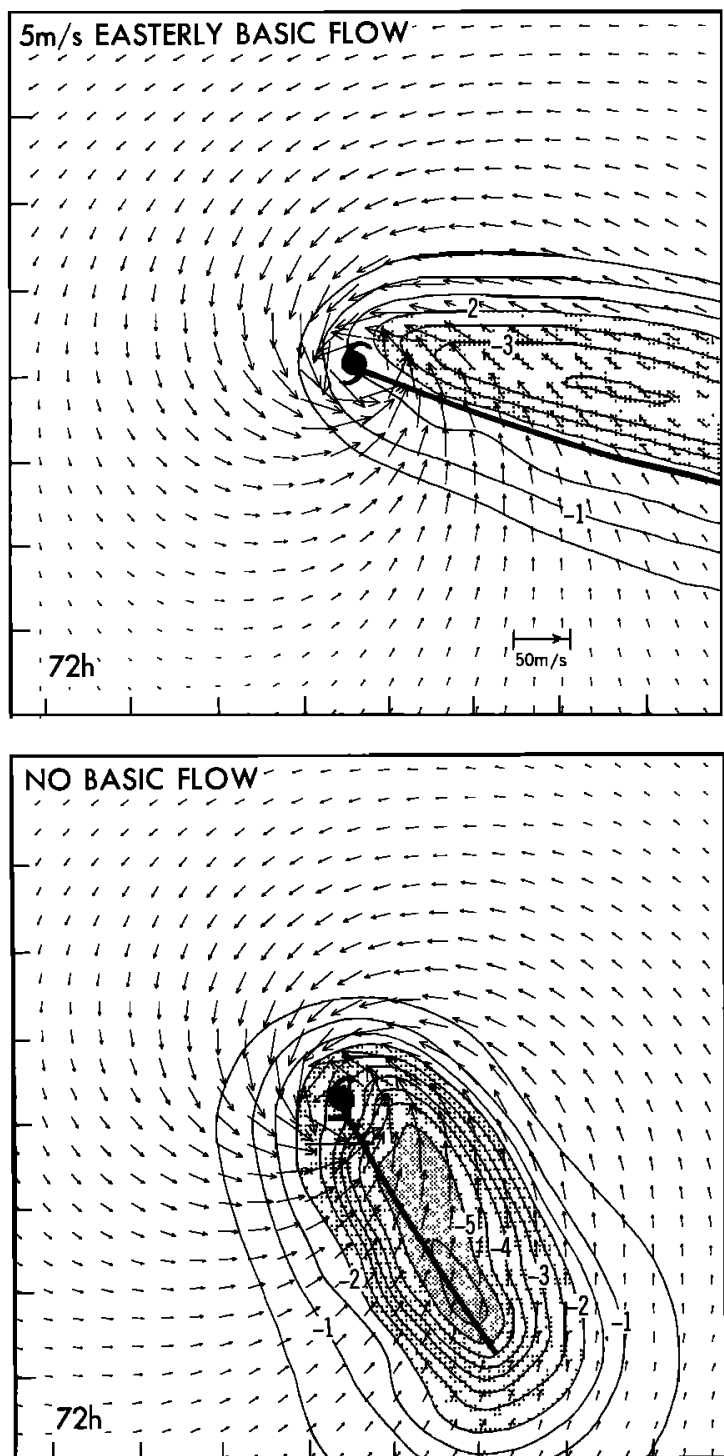

Fig. 5. The SST anomalies $\left({ }^{\circ} \mathrm{C}\right)$ and wind vectors of the lowlevel $(\sigma=0.995)$ wind at 72 hours for the coupled experiments with the $5 \mathrm{~m} \mathrm{~s}^{-1}$ easterly basic flow (top) and no basic flow (bottom). The area shown is for the region of the innermost nest, with the tick marks drawn at $1^{\circ}$ intervals. The regions with anomalies larger than $2^{\circ}$ and $5^{\circ}$ are indicated with different shading. The hurricane center at 72 hours is indicated by the hurricane symbol with the storm track indicated by the solid line.
HEAT FLUXES
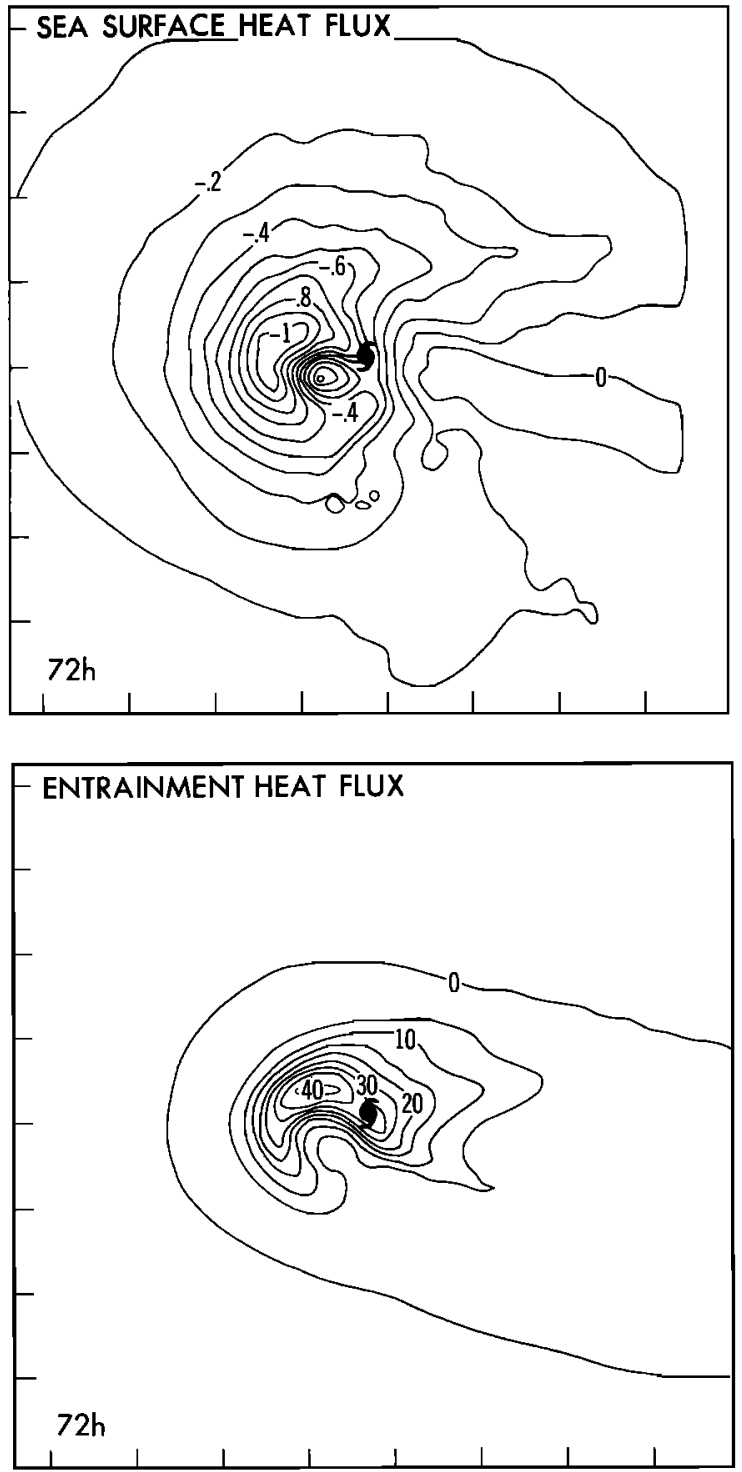

Fig. 6. The distribution of the total surface heat flux $\left(\mathrm{kW} \mathrm{m}^{-2}\right)$ (positive value directed downward into the ocean) and entrainment heat flux at the bottom of the ocean mixed layer at 72 hours for the coupled experiment with the $5 \mathrm{~m} \mathrm{~s}^{-1}$ easterly basic flow. The area shown is for the region of the ocean grid covered by the innermost nest of the hurricane model. The tick marks are drawn at $1^{\circ}$ intervals. The hurricane center at 72 hours is indicated by the hurricane symbol.

ated. Most of the discussion will concentrate on the experiments run with the easterly basic flows, which are more typical for storms which occur over the western Atlantic basin in the deep tropics.

\subsection{Changes in Surface Heat Flux}

As previously mentioned, all experiments were integrated to 72 hours. The cooling of the sea surface in Figure 5 produced a significant reduction of total heat flux (latent plus sensible) into the hurricane (Figure 7). For example, with the $5 \mathrm{~m} \mathrm{~s}^{-1}$ easterly basic flow the maximum total heat flux averaged for the entire integration period decreased from $1.25 \mathrm{~kW} \mathrm{~m}^{-2}$ in the control experiment to about $0.9 \mathrm{~kW} \mathrm{~m}^{-2}$ in the coupled experiment. In the latter integration the 


\section{$5 \mathrm{~m} / \mathrm{s}$ EASTERLY BASIC FLOW TOTAL HEAT FLUX}
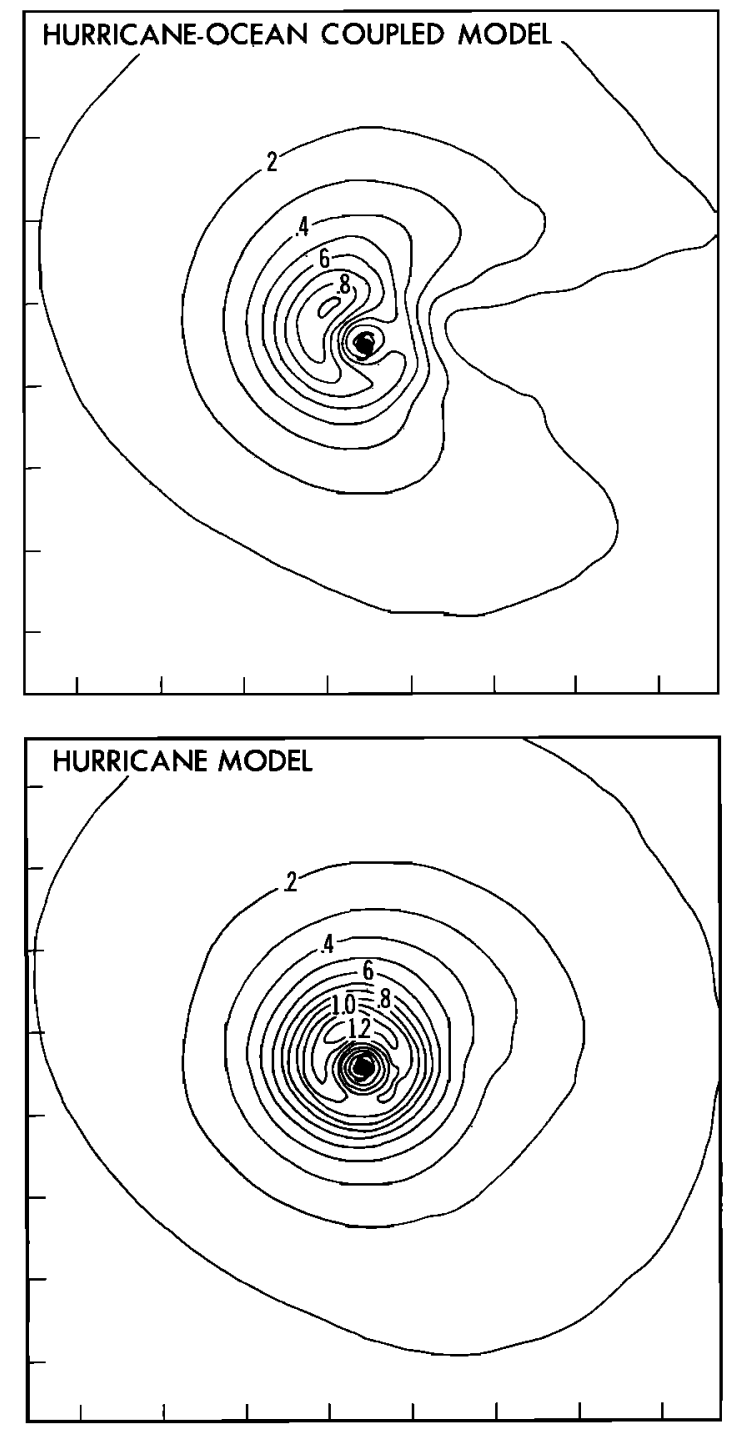

Fig. 7. The distribution of the total surface heat flux $\left(\mathrm{kW} \mathrm{m}^{-2}\right)$ (positive value directed upward into the atmosphere) averaged for the entire $\mathbf{7 2}$ hours of the integration and computed relative to the moving storm for the experiments run both with (top) and without (bottom) ocean coupling. The area shown is for the region of the innermost nest, with the tick marks drawn at $1^{\circ}$ intervals. The hurricane center position is indicated by the hurricane symbol.

pronounced asymmetry in the distribution of this quantity is very evident with the heat flux greatly reduced in the regions above the cold wake. The primary contribution to the decrease in the total heat flux in these two experiments was the reduction in the latent heat flux. When averaged over the entire region in Figure 7 (nest 3) the latent heat flux decreased from 0.22 to $0.17 \mathrm{~kW} \mathrm{~m}^{-2}$. Likewise, the value of the average sensible heat flux for the control and the coupled experiment in this region changed from -0.0058 to -0.011 $\mathrm{kW} \mathrm{m} \mathrm{m}^{-2}$ as more sensible heat was transported from the atmosphere into the area of the reduced SST.

The decrease of the total heat flux due to the ocean coupling can be seen clearly in Figure 8 for the set of experiments with the $5 \mathrm{~m} \mathrm{~s}^{-1}$ easterly basic flow and no basic flow. For both cases the asymmetry in the reduction of heat flux is evident. Over the cold wake the reduction was considerable and was greater for the slower-moving storm where the ocean response was larger. For the experiment with no basic flow the region of SST anomalies greater than $2^{\circ}$ (stippled area in Figure 5) extended over the central area of the storm resulting in a decrease of the total heat flux of over $1.0 \mathrm{~kW} \mathrm{~m} \mathrm{~m}^{-2}$ in the eye wall region of the storm where the heat flux maximum was located. For these two sets of experiments the value of the maximum total heat flux decreased nearly $30 \%$ and $60 \%$, respectively.

In accordance with the reduction of the total heat flux the total storm precipitation accumulated relative to the moving storm significantly decreased (Figure 9) for both sets of

\section{DECREASE OF HEAT FLUXES DUE TO OCEAN COUPLING}
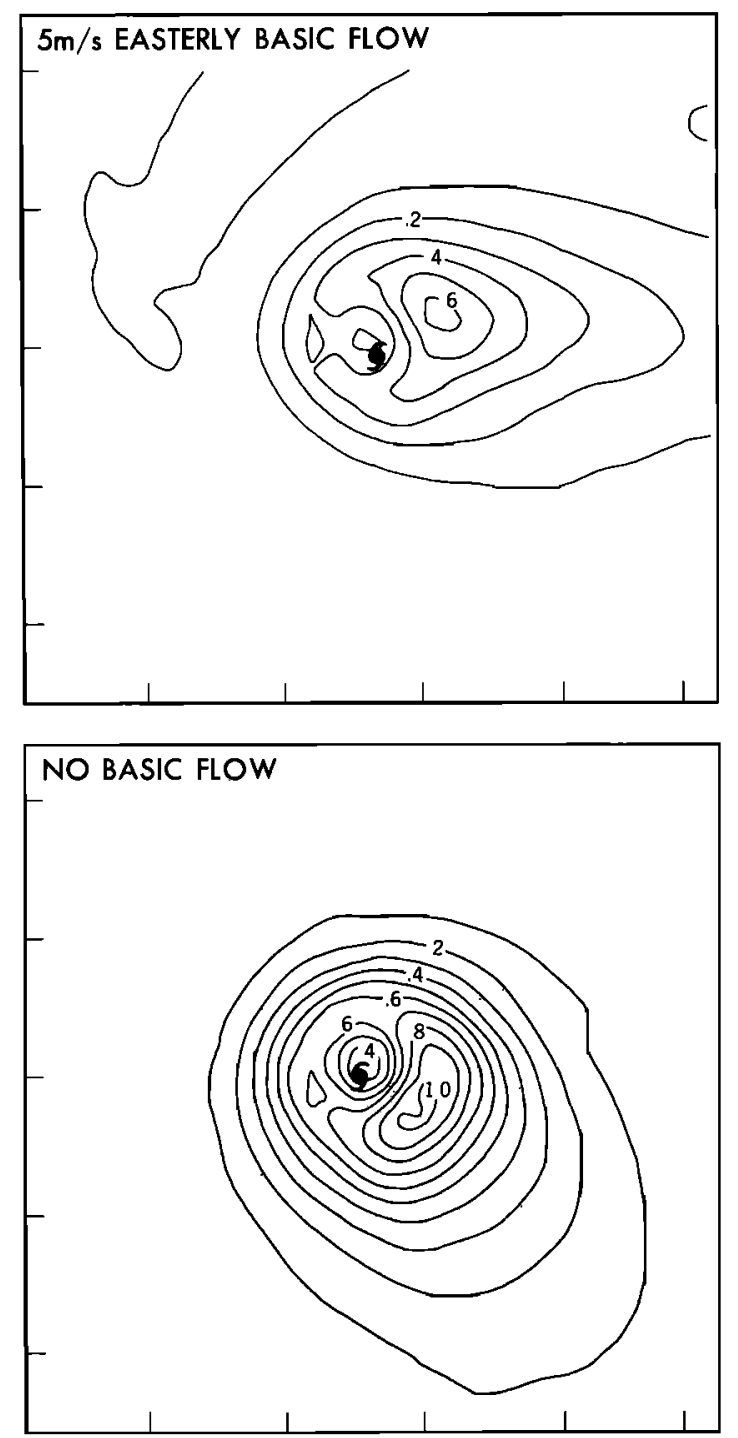

Fig. 8. The decrease of the total surface heat flux $\left(\mathrm{kW} \mathrm{m}^{-2}\right)$ between the coupled and the noncoupled experiments averaged for the entire 72 hours of the integration and computed relative to the moving storm for both the $5 \mathrm{~m} \mathrm{~s}^{-1}$ easterly basic flow (top) and the no basic flow (bottom) experiments. The region shown is for the center portion of the innermost nest. The hurricane center position is indicated by the hurricane symbol. 


\section{DECREASE OF PRECIPITATION DUE TO OCEAN COUPLING}
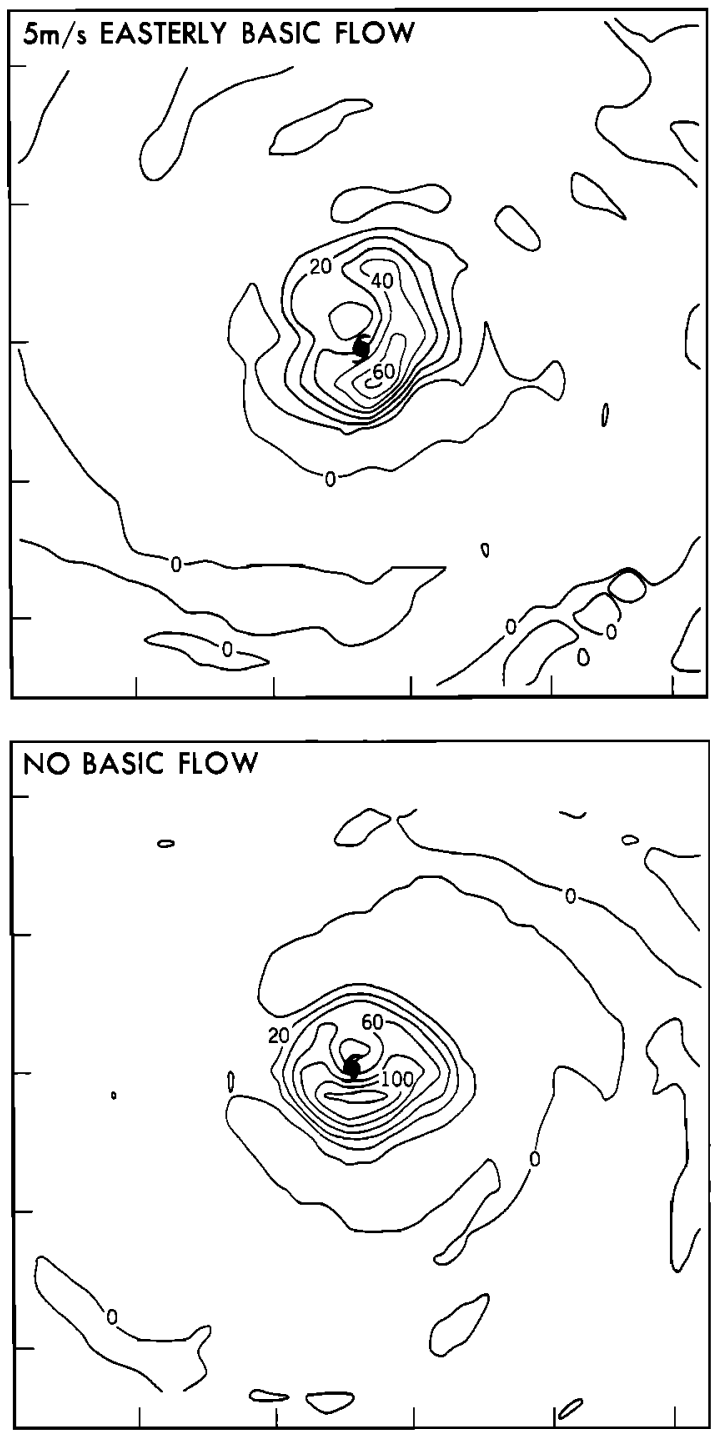

Fig. 9. The decrease in the total storm precipitation (centimeters) between the coupled and the noncoupled experiments, accumulated relative to the moving storm for the entire 72 hours of the integration for both the $5 \mathrm{~m} \mathrm{~s}^{-1}$ easterly basic flow (top) and the no basic flow (bottom) experiments. The region shown is for the center portion of the innermost nest with the tick marks drawn at $1^{\circ}$ intervals. The hurricane center position is indicated by the hurricane symbol.

coupled experiments. The large decrease was located in the eye wall region with the position of the maximum decrease in precipitation appearing to be biased toward the quadrant of the storm where the largest decrease in heat flux occurred. For example, for the integrations with the $5 \mathrm{~m} \mathrm{~s}^{-1}$ easterly basic flow, the precipitation was reduced between 10 and $20 \%$ (maximum decrease of about $60 \mathrm{~cm}$ ) on the eastern side of the storm center over the cold wake in contrast to only 5 to $10 \%$ (maximum decrease of about $20 \mathrm{~cm}$ ) on the western side where the reduction of heat fluxes due to the ocean coupling was much smaller. For the set of experiments with no basic flow the reduction of precipitation in the eye wall region south of the storm center ranged between 20 and $50 \%$ (maximum decrease of over $120 \mathrm{~cm}$ ) and between 5 and $20 \%$ (maximum less than $60 \mathrm{~cm}$ ) north of the storm.

Another feature produced by the decrease of the heat flux due to the ocean coupling is seen in the horizontal (Figure 10) and the vertical (Plate 1) distributions of the equivalent potential temperature. In the regions over the cold wake underneath and to the east of the storm center the equivalent potential temperature in the lower boundary layer decreased by about $2^{\circ}$ to $6^{\circ}$ (Figure $10 \mathrm{c}$ ) relative to the noncoupled experiment. Although the area of the cold wake was rather narrow (Figure 5), the effect on the equivalent potential temperature spread over a large region east and north of the storm center (Figure 10). The decrease of the equivalent potential temperature was generally much smaller in the region west and south of the storm. Apparently, the necessary heat energy to drive the storm was provided by the warm moist air from this region. However, the equivalent potential temperature at the storm center was significantly less than the value attained in the noncoupled experiment. Therefore as seen in both Figure 10 and Plate 1, the gradient of the equivalent potential temperature in the center area was considerably weakened with the ocean coupling, particularly in the lower boundary layer. The difference of the equivalent potential temperature between the storm center and the outer storm periphery $(\sim 450 \mathrm{~km})$, calculated in the lowest $500 \mathrm{~m}$ of the boundary layer and averaged for all four sides of the storm, decreased from $16^{\circ}$ to $12^{\circ}$ due to the ocean coupling. These values correlated reasonably well with the average sea level pressure difference of about 70 and $55 \mathrm{hPa}$, respectively (measured between the storm center and the outer storm periphery), for the noncoupled and coupled experiments.

From Plate 1 it is clearly seen that the resulting reduction in the moist static energy in the boundary layer directly affected the warm core temperature. The upper level equivalent potential temperature was lowered nearly $4^{\circ}$ compared to the noncoupled case. In comparison, for the experiment with no basic flow (figure not shown) the decrease exceeded $7^{\circ}$. The decrease with height of the equivalent potential temperature observed within the model eye between the boundary layer and the 4-km level in both the coupled and the noncoupled experiments may have been related to subsidence and horizontal mixing of the air within the eye region [e.g., Kurihara and Bender, 1982].

\subsection{Changes in Storm Intensity}

The impact of the ocean coupling on the storm intensity is demonstrated in the time series of minimum sea level pressure (Figure 11) and maximum low level winds (Figure 12) for each of the cases with the easterly basic flow. During the first 12 hours, as the storm was undergoing rapid strengthening, the effect on storm intensification was relatively small. However, after 12 hours without ocean interaction the storms continued to rapidly intensify, especially in the cases with weaker basic flows. In contrast, for all of the coupled experiments the intensification rate began to level off after 12 hours. In the experiments with the faster basic flows ( 5 and $7.5 \mathrm{~m} \mathrm{~s}^{-1}$ ) the impact of the ocean interaction on storm intensity remained small until around 30 and 36 hours, respectively. Beyond this time the storm intensity increased only slightly in the coupled experiments, while significant storm intensification (about $15 \mathrm{hPa}$ deepening and $6 \mathrm{~m} \mathrm{~s}^{-1}$ 
EQUIVALENT POTENTIAL TEMPERATURE $(5 \mathrm{~m} / \mathrm{s}$ BASIC FLOW)
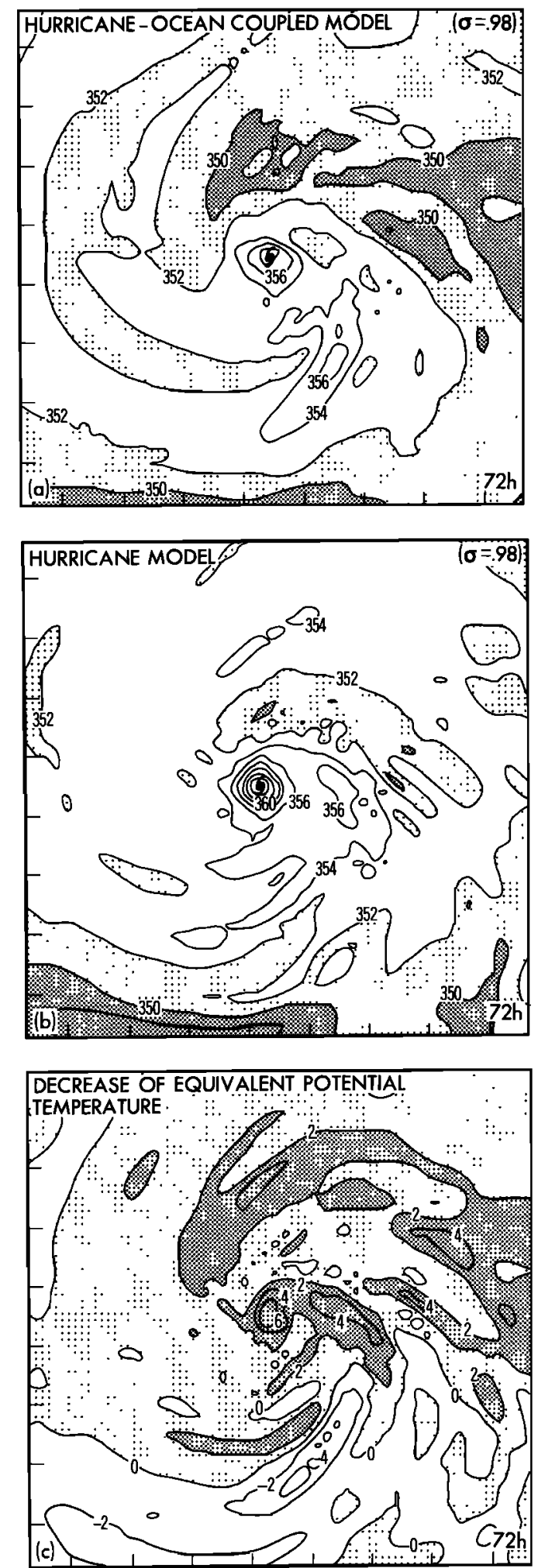

Fig. 10. Distribution at 72 hours of the model level $17(\sigma=0.98)$ equivalent potential temperature $\left({ }^{\circ} \mathrm{K}\right)$ for the $5 \mathrm{~m} \mathrm{~s}^{-1}$ basic flow experiments run $(a)$ with and $(b)$ without ocean coupling as well as (c) the decrease of the equivalent potential temperature between the coupled and the noncoupled experiments. The contour interval is $2^{\circ}$, with values less than $352^{\circ}$ and $350^{\circ}$ in Figures $10 a$ and $10 b$ and values greater than $0^{\circ}$ and $2^{\circ}$ in Figure $10 c$, indicated by lighter and thicker shading, respectively. See Figure 6 for more details. increase of the low-level winds) occurred during the remaining 36 hours of the noncoupled integrations.

The time series of the maximum SST decrease are presented in Figure 13. Comparison of Figures 11, 12, and 13 indicate that the greatest intensity differences between the coupled and the noncoupled models occurred in the experiments with the weaker basic flows where the greatest decrease of the SST occurred and the decrease of heat flux (Figure 8) was greatest. For example, at 72 hours the maximum SST cooling was $5.9^{\circ} \mathrm{C}$ for the no basic flow case and about $3.5^{\circ} \mathrm{C}$ for the $5 \mathrm{~m} \mathrm{~s}^{-1}$ easterly flow. The differences from the control experiments at 72 hours in the minimum sea level pressure and maximum surface winds for the former case was about $20 \mathrm{hPa}$ and $-8 \mathrm{~m} \mathrm{~s}^{-1}$ compared to $14 \mathrm{hPa}$ and $-5 \mathrm{~m} \mathrm{~s}^{-1}$ for the latter. This result was consistent with other earlier numerical studies performed with a much simpler experimental design [e.g., Khain and Ginis, 1991; Sutyrin and Khain, 1984].

The average differences in minimum sea level pressure, maximum low-level wind, and maximum SST cooling are summarized in Table 3, calculated for the final 48 hours of the integrations for all sets of experiments performed. The average translational speed for each storm during this period is also included in the second column. The storms embedded in the westerly basic flows moved slower than those with corresponding easterly flow since the basic flow in these cases was oriented in a direction almost opposite to the flow due to the beta gyre. For example, the average storm translational speed during this period was $6.1 \mathrm{~m} \mathrm{~s}^{-1}$ for the storm embedded in the $5 \mathrm{~m} \mathrm{~s}^{-1}$ easterly basic flow and $4.2 \mathrm{~m}$ $\mathrm{s}^{-1}$ for the storm in the $5 \mathrm{~m} \mathrm{~s}^{-1}$ westerly basic flow. This resulted in larger sea surface cooling with the storms moving in the westerly basic flow and a greater decrease in intensity for these cases. A strong correlation was found between the maximum sea surface cooling and the changes in storm intensity for the cases with both the easterly and the westerly moving storms. A conclusion can now be made from these results that the faster-moving storms produced a progressively smaller SST response and smaller reduction in tropical cyclone strength measured by both the minimum sea level pressure and the maximum low-level winds.

Examples of observed maximum sea surface cooling for tropical cyclones in various regions are summarized in Table 4. All of these storms were of either moderate or strong hurricane intensity, similar to the storms in our experiments. The observed maximum cooling varied from $1^{\circ}$ to $6^{\circ}$ in good agreement with the model results. The observations were grouped according to slow (less than $4 \mathrm{~m} \mathrm{~s}^{-1}$ ), medium (between 4 and $8 \mathrm{~m} \mathrm{~s}^{-1}$ ), and fast (greater than $8 \mathrm{~m} \mathrm{~s}^{-1}$ ) translational speeds. This yielded average cooling for the three groups of $5.3^{\circ}, 3.5^{\circ}$, and $1.8^{\circ}$, respectively. For our numerical experiments the same grouping of storm motion yielded values of $4.8^{\circ}, 3.3^{\circ}$, and $2.6^{\circ}$. Although these observed ocean responses were probably sensitive to the specific vertical profiles of the ocean stratification, this data set clearly showed that the magnitude of the simulated sea surface cooling was reasonable and that the obtained relationship between the sea surface cooling and the storm motion for the numerical simulations was similar to that found in the observational data.

It should be pointed out that in the noncoupled experiments with easterly flow, the storms progressively became more intense with the smaller basic flows (Figures 11 and 

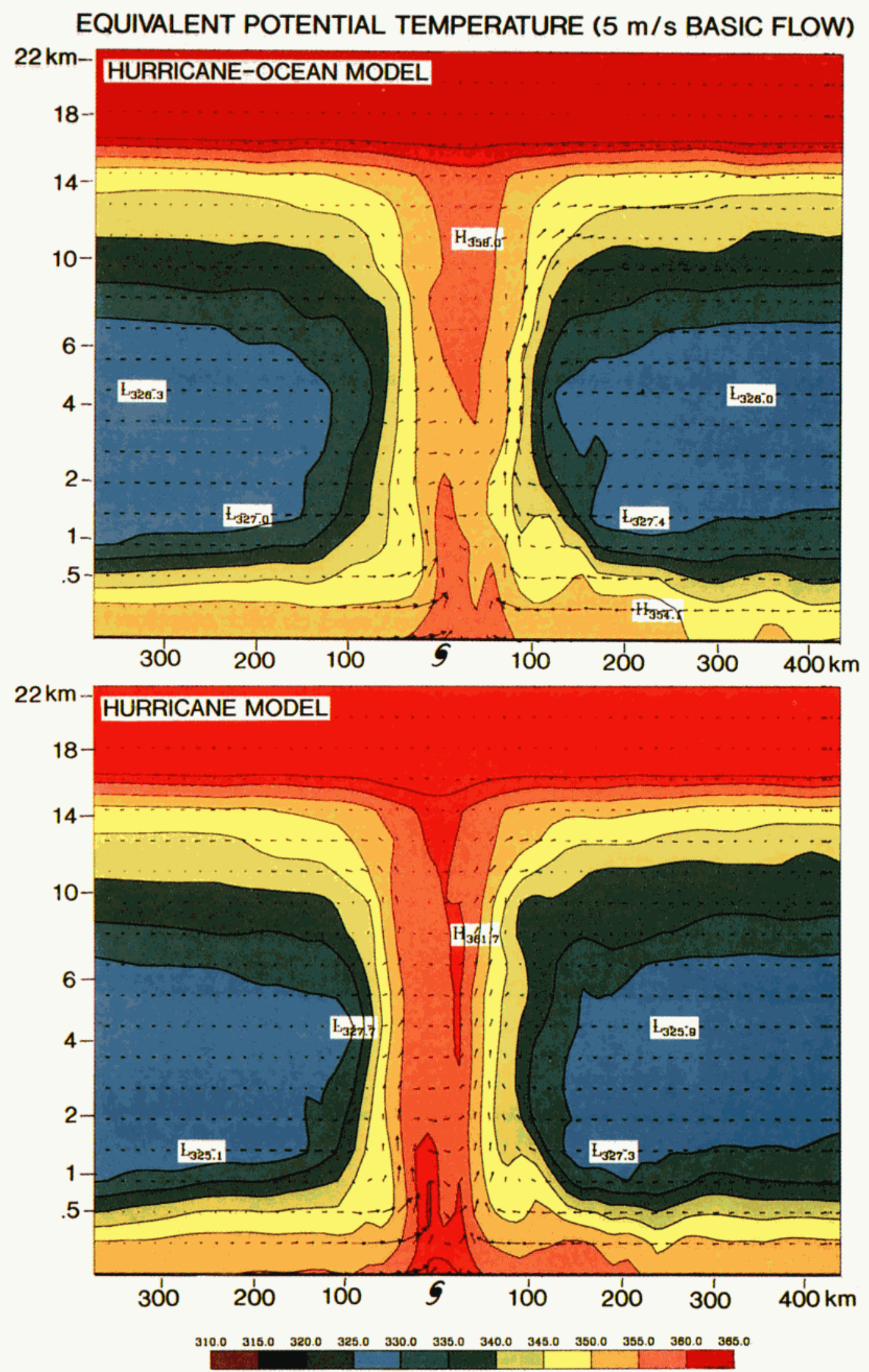

Plate 1. East-west cross section through the storm center of the equivalent potential temperature $\left({ }^{\circ} \mathrm{K}\right)$ at 72 hours for the $5 \mathrm{~m} \mathrm{~s}^{-1}$ basic flow experiments run with (top) and without (bottom) ocean coupling. The contour interval is $5^{\circ}$ with no additional contours drawn for the area of equivalent potential temperature above $365^{\circ}$. The vertical coordinate is the square root of height in meters. (See Figure 11 of Bender et al. [1985] for more details of this coordinate system). The arrows plotted at each grid point are the wind vectors. 

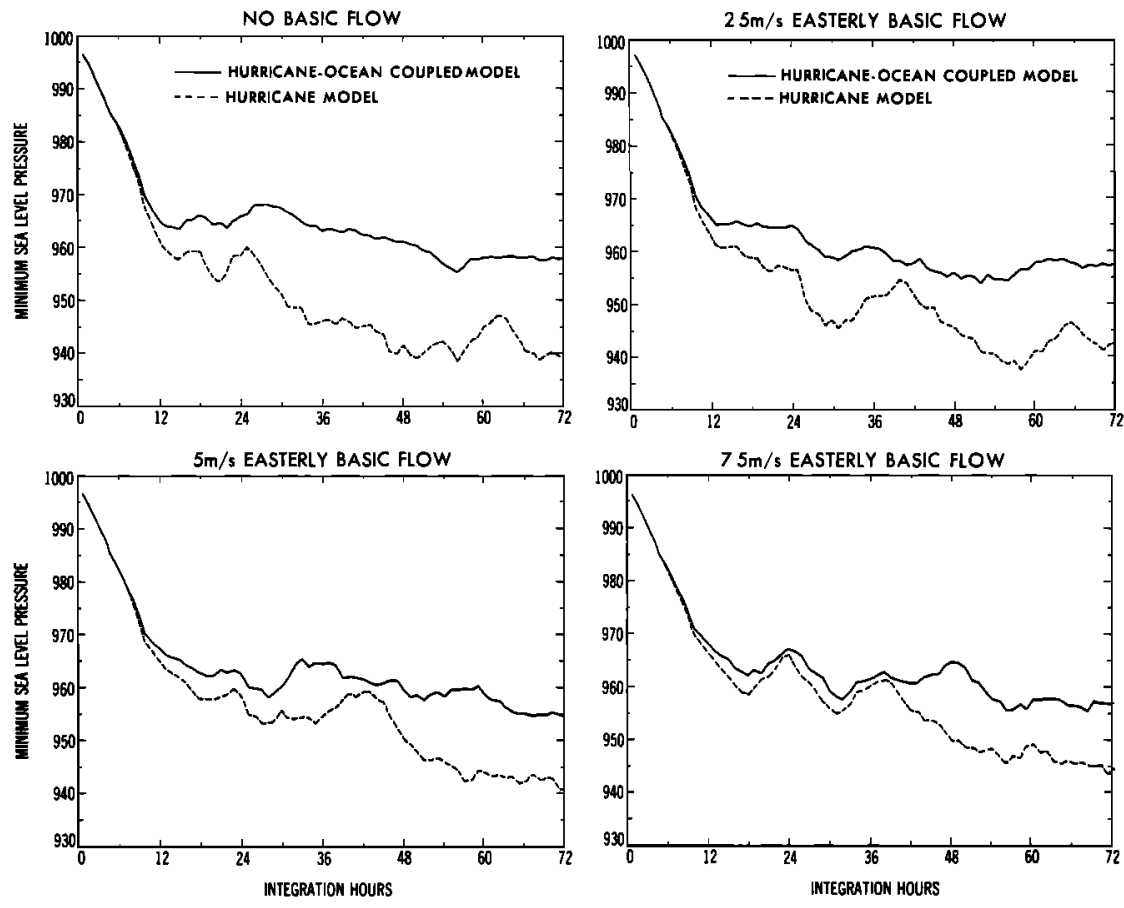

Fig. 11. Time series of minimum sea level pressure $(\mathrm{hPa})$ for the no basic flow experiments and all experiments with easterly basic flows run with hurricane-ocean coupling (solid curve) and without coupling (dashed curve).

12). Hence the differences in the reduction in intensity shown in Table 3 between the faster-moving and the slowmoving storms with easterly basic flow were primarily a result of differences in storm strength between the noncoupled control experiments. With the coupling included, the average difference in the minimum sea level pressure during the final 12 hours of the integrations varied by only about 3 $\mathrm{hPa}$ between the no basic flow $(958 \mathrm{hPa})$ and the $7.5 \mathrm{~m} \mathrm{~s}^{-1}$ $(955 \mathrm{hPa})$ basic flow experiments. Analysis of the kinetic energy budgets for the noncoupled experiments indicated that without the basic flow the divergence of kinetic energy away from the storm region remained very small in the
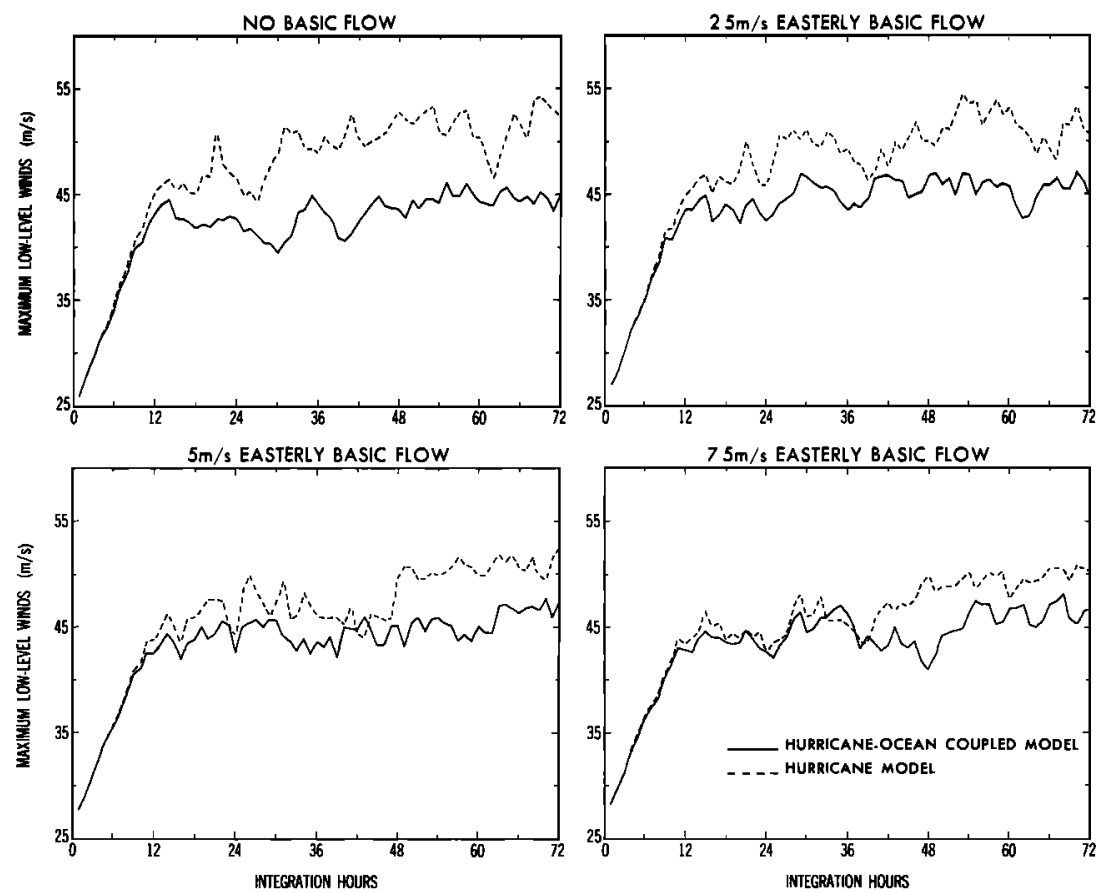

Fig. 12. Time series of maximum low-level $(\sigma=0.995)$ winds $\left(\mathrm{m} \mathrm{s}^{-1}\right)$ for the no basic flow experiments and all experiments with easterly basic flows run with hurricane-ocean coupling (solid curve) and without coupling (dashed curve). 


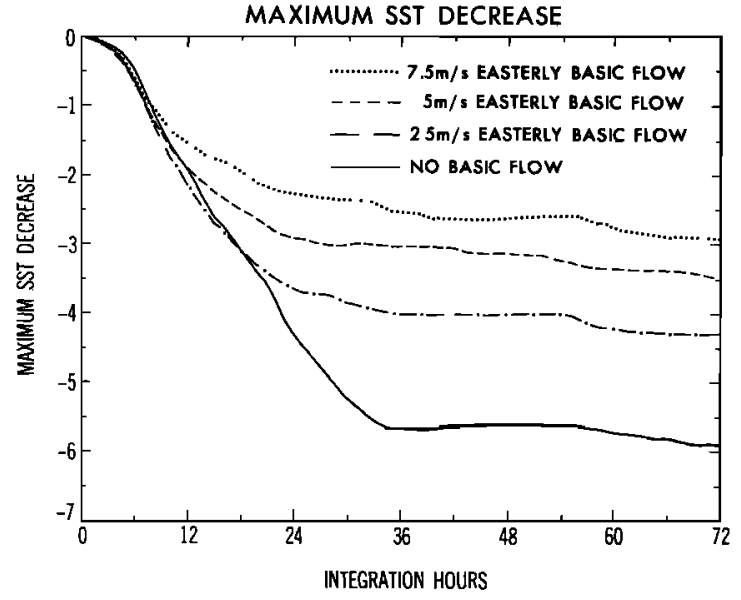

Fig. 13. Time series of maximum SST decrease $\left({ }^{\circ} \mathrm{C}\right)$ during the 72-hour coupled integrations for the three cases with the easterly basic flow and the case with no initial basic flow.

middle atmosphere (between the 850 - and the $250-\mathrm{hPa}$ level). However, in the presence of easterly basic flow (both with and without coupling) a divergence of kinetic energy took place between the 900 - and the $800-\mathrm{hPa}$ levels and also between the 500 - and the $200-\mathrm{hPa}$ levels. This effect may have contributed to this systematic decrease in the storm intensity in these noncoupled experiments with the inclusion of the basic flows. However, the relationship between storm intensity and environmental flow is not fully understood. Indeed, for the noncoupled integrations with westerly basic flow a similar correlation was not evident between storm intensity and magnitude of the basic flow. Once the ocean coupling was included, the slower-moving storms in these integrations became considerably weaker than those embedded in the stronger westerly flows. The minimum sea level pressure at 72 hours was about $8 \mathrm{hPa}$ higher in the no basic flow experiment compared with the $7.5 \mathrm{~m} \mathrm{~s}^{-1}$ westerly flow experiment.

In previous numerical experiments with hurricane-ocean coupled models [e.g., Khain and Ginis, 1991; Sutyrin and
Khain, 1984] the model tropical cyclones responded with about a 1-day delay to the decrease of the SST. However, as mentioned before, these earlier experiments were carried out with both a simplified experimental design and a coarse grid resolution (e.g., 40 and $60 \mathrm{~km}$ ). Also, the intensity of the tropical cyclones [e.g., Khain and Ginis, 1991, $1000 \mathrm{hPa}$ was considerably weaker at the time the ocean-coupling began. To test the response of a mature tropical cyclone to the effect of the ocean coupling, a supplemental experiment was performed for the $5 \mathrm{~m} \mathrm{~s}^{-1}$ easterly flow case in which the ocean coupling began at 24 hours (Figure 14). By this time the minimum sea level pressure of the storm had dropped to $955 \mathrm{hPa}$. As seen in Figure 14 (bottom) the ocean immediately began to respond to the effect of the tropical cyclone as the SST in the eye wall region began to rapidly decrease. Within 12 hours the maximum SST anomaly reached the same value as the original coupled experiment. Likewise, (Figure 14, top) the tropical cyclone also began to immediately respond to the ocean coupling with a rise in the minimum sea level pressure. The minimum sea level pressure of the tropical cyclone reached nearly an identical value as the original coupled experiment 12 hours after the coupling of the two models began. Both storms remained at nearly the same intensity for the duration of the integrations. This clearly demonstrates that for these idealized experiments in which other environmental effects such as vertical wind shear were absent, the final equilibrium states of the tropical cyclones were primarily determined by the SST.

Finally, it should also be noted that even in the experiment performed without the basic flow and ocean coupling, the minimum sea level pressure $(939 \mathrm{hPa})$ obtained by the model tropical cyclone was somewhat higher than the potential minimum computed from either the initial (Figure $2 a$ ) or the 48-hour environmental sounding of the matured storm (928 or $914 \mathrm{hPa}$, respectively) using the formulation of Emanuel [1988]. Probably, the finest resolution used in the present experiments was still not sufficient to adequately resolve the structure of the eye or eyewall which may have reduced the model storm intensity. Also, the treatment of convection and radiation could have influenced the storm intensity in the numerical model.

TABLE 3. Differences Between the Coupled and the Noncoupled Models Averaged for the Final 48 hours of the Integrations Presented in This Study

\begin{tabular}{|c|c|c|c|c|}
\hline \multirow[b]{2}{*}{$\begin{array}{l}\text { Basic Flow, } \\
\mathrm{m} \mathrm{s}^{-1}\end{array}$} & \multirow[b]{2}{*}{$\begin{array}{l}\text { Translational } \\
\text { Speed, } \\
\text { m s }^{-1}\end{array}$} & \multicolumn{3}{|c|}{ Coupled-Noncoupled } \\
\hline & & $\begin{array}{l}\text { Minimum Sea } \\
\text { Level Pressure } \\
\text { Difference, hPa }\end{array}$ & $\begin{array}{l}\text { Maximum } \\
\text { Surface Wind } \\
\text { Difference, } \\
\mathrm{m} \mathrm{s}^{-1}\end{array}$ & $\begin{array}{c}\text { Maximum SST } \\
\text { Decrease, }{ }^{\circ} \mathrm{C}\end{array}$ \\
\hline & & No Basic Flow & & \\
\hline & 1.7 & 16.4 & -7.0 & -5.6 \\
\hline $\begin{array}{l}2.5 \\
5.0 \\
7.5\end{array}$ & $\begin{array}{l}2.2 \\
4.2 \\
6.6\end{array}$ & $\begin{array}{c}\text { Westerly Flow } \\
15.6 \\
12.0 \\
7.0\end{array}$ & $\begin{array}{l}-6.7 \\
-4.8 \\
-2.6\end{array}$ & $\begin{array}{l}-4.6 \\
-3.7 \\
-3.0\end{array}$ \\
\hline $\begin{array}{l}2.5 \\
5.0 \\
7.5\end{array}$ & $\begin{array}{l}3.8 \\
6.1 \\
8.3\end{array}$ & $\begin{array}{c}\text { Easterly Flow } \\
11.8 \\
9.7 \\
7.7\end{array}$ & $\begin{array}{l}-5.0 \\
-3.7 \\
-2.8\end{array}$ & $\begin{array}{l}-4.1 \\
-3.2 \\
-2.6\end{array}$ \\
\hline
\end{tabular}

The average translational speed for each storm is also included in column 2. SST, sea surface temperature. 
TABLE 4. Maximum Sea Surface Cooling Observed After the Passage of 16 Tropical Cyclones (of Moderate or Strong Intensity) in Various Regions

\begin{tabular}{|c|c|c|c|c|}
\hline $\begin{array}{c}\text { Tropical } \\
\text { Cyclone Storm } \\
\text { Name, Year }\end{array}$ & $\begin{array}{c}\text { Storm } \\
\text { Motion, } \\
\mathrm{m} \mathrm{s}^{-1}\end{array}$ & Region & $\begin{array}{l}\text { Maximum } \\
\text { Cooling, } \\
{ }^{\circ} \mathrm{C}\end{array}$ & Reference \\
\hline \multicolumn{5}{|c|}{ Slow-Moving Storms (Less Than $4 \mathrm{~m} \mathrm{~s}^{-1}$ ) } \\
\hline Virginia, 1978 & 1.5 & western North Pacific & 6.0 & Pudov [1980] \\
\hline Ella, 1978 & 2.0 & western Atlantic & 4.5 & Black [1983] \\
\hline Hilda, 1964 & 3.0 & Gulf of Mexico & 6.0 & Leipper [1967] \\
\hline Carla, 1961 & 3.0 & Gulf of Mexico & 4.8 & Stevenson and Armstrong [1965] \\
\hline Average & 2.4 & & 5.3 & \\
\hline \multicolumn{5}{|c|}{ Medium-Moving Storms (Between 4 and $8 \mathrm{~m} \mathrm{~s}^{-1}$ ) } \\
\hline Joan, 1970 & 4.0 & South China Sea & 4.0 & Ramage et al. [1974] \\
\hline Norbert (1984) & 4.3 & eastern North Pacific & 2.2 & Sanford et al. [1987] \\
\hline Tess (1975) & 6.0 & western North Pacific & 4.0 & Pudov et al. [1978] \\
\hline Harvey (1981) & 6.0 & Sargasso Sea & 3.5 & Stramma et al. [1986] \\
\hline Gay (1985) & 6.0 & western North Pacific & 2.0 & Church et al. [1989] \\
\hline Gilbert (1988) & 6.5 & Gulf of Mexico & 4.0 & Shay et al. [1991] \\
\hline Gloria (1985) & 6.8 & $\begin{array}{l}\text { western North } \\
\text { Atlantic }\end{array}$ & 5.0 & Cornillon et al. [1987] \\
\hline Average & 5.7 & & 3.5 & \\
\hline \multicolumn{5}{|c|}{ Fast-Moving Storms (Greater Than $8 \mathrm{~m} \mathrm{~s}^{-1}$ ) } \\
\hline Eloise (1975) & 9.0 & Gulf of Mexico & 2.5 & Johnson and Withee [1978] \\
\hline Phyllis (1975) & 10.0 & western North Pacific & 2.0 & Schramm [1979] \\
\hline Shirley (1965) & 13.0 & Japan east coast & 2.5 & Wright [1969] \\
\hline Belle (1976) & 13.0 & West Atlantic & 1.0 & Johnson and Speer [1978] \\
\hline Ida (1958) & 15.0 & western North Pacific & 1.0 & Ogata $[1960]$ \\
\hline Average & 12.0 & & 1.8 & \\
\hline
\end{tabular}

The storms are grouped according to their translational speed.

\subsection{Changes in Storm Track}

To evaluate the effect of the tropical cyclone-ocean coupling on the storm motion, the storm tracks were analyzed for each set of experiments. Figure 15 shows the storm tracks in the experiments with both easterly and westerly basic flows of $2.5 \mathrm{~m} \mathrm{~s}^{-1}$. A small effect on the storm track was introduced by the ocean coupling particularly with the easterly basic flow. After 36 hours the storm turned slightly more to the north and was located about $45 \mathrm{~km}$ to the north of the noncoupled experiment by 72 hours. For the storms with the 5 and $7.5 \mathrm{~m} \mathrm{~s}^{-1}$ easterly and westerly flows the difference in the storm tracks remained extremely small throughout the entire integration. An example is shown in Figure 16 (bottom) for the $7.5 \mathrm{~m} \mathrm{~s}^{-1}$ easterly basic flow. It is also evident that the largest impact on the storm track occurred with no basic flow (Figure 16, top). In this experiment the storm with the ocean interaction gradually turned more to the north and to the east by the end of the second day of the integration and was located over $70 \mathrm{~km}$ to the east-southeast of the noncoupled experiment by 72 hours. A possible explanation for this track deviation is related to a systematic weakening of the circular averaged tangential flow at all radii of the storm induced by the interaction with the ocean (Figure 17). This effect was greatest in the experiments with no basic flow where decreases of nearly $2 \mathrm{~m} \mathrm{~s}^{-1}$ in the winds in the outer radii of the storm were found. Although these differences were relatively small, they were probably sufficient to have altered the orientation of the beta gyre and thus affected the beta drift. Fiorino and Elsberry [1989] found that differences of about $2 \mathrm{~m} \mathrm{~s}^{-1}$ in the tangential wind profiles in the 300 - to $700-\mathrm{km}$ radius of an idealized vortex resulted in similar changes in storm track in a nondivergent, barotropic numerical model with no basic current. Differences in storm motion presented in these results and those obtained by Khain and Ginis [1991] probably resulted from differences in the impact of the coupling on the beta gyre between the two sets of experiments, due to the different experimental design and grid resolution. For the experiments with the 7.5 and $5 \mathrm{~m} \mathrm{~s}^{-1}$ basic flow where no significant track deflection occurred, the decrease of the winds in the outer radius was less than $1 \mathrm{~m} \mathrm{~s}^{-1}$.

It is interesting to note in Figure 16 that in the $7.5 \mathrm{~m} \mathrm{~s}^{-1}$ easterly basic flow experiment there was a tendency for the storm with the ocean interaction to move slightly faster than the noncoupled case. A similar response occurred in the 7.5 $\mathrm{m} \mathrm{s}^{-1}$ westerly basic flow experiment. One possible mechanism that may be responsible for this slight increase of forward speed was the large reduction of precipitation that occurred to the rear of the storm shown in Figure 9. This asymmetric decrease of precipitation on one side of the storm may have introduced a slight shift in the surface pressure minimum toward the region of stronger condensational heating which in both of these experiments was located in front of the storm.

\section{Summary and Concluding Remarks}

The effect of tropical cyclone-ocean interaction was investigated with a high-resolution tropical cyclone-ocean coupled model. The model was formed by coupling the GFDL 18-level movable-mesh tropical cyclone prediction model with an 8-layer primitive equation ocean model. The resolution of the inner nest of the atmospheric model as well as the uniform resolution of the ocean model was $1 / 6^{\circ}$. Thus the vertical and horizontal resolutions of the coupled model were considerably finer than those used by previous numer- 
ical models that have investigated the interaction of the tropical cyclone-ocean system. In the present experiments the hurricane was embedded in easterly and westerly environmental flows of $2.5,5.0$, and $7.5 \mathrm{~m} \mathrm{~s}^{-1}$. For comparison, similar experiments were carried out without coupling (control cases). The initial tangential wind profile of the tropical cyclone used was similar to the observed profile for Hurricane Gloria just after it was first upgraded to a hurricane. A supplemental experiment was also performed in which no basic flow was included, and hence the tropical cyclone movement was due entirely to the beta effect. This supplemental integration was designed to simulate those situations where tropical cyclones are embedded in weak steering flows.

The numerical results demonstrated that the ocean coupling produced a significant cooling of the sea surface, decrease of the total heat flux directed into the hurricane from the ocean, and hence reduction in hurricane intensity. The storm weakening was consistently greater for the slower moving storms where the SST decrease was larger. A general conclusion from both the easterly and the westerly
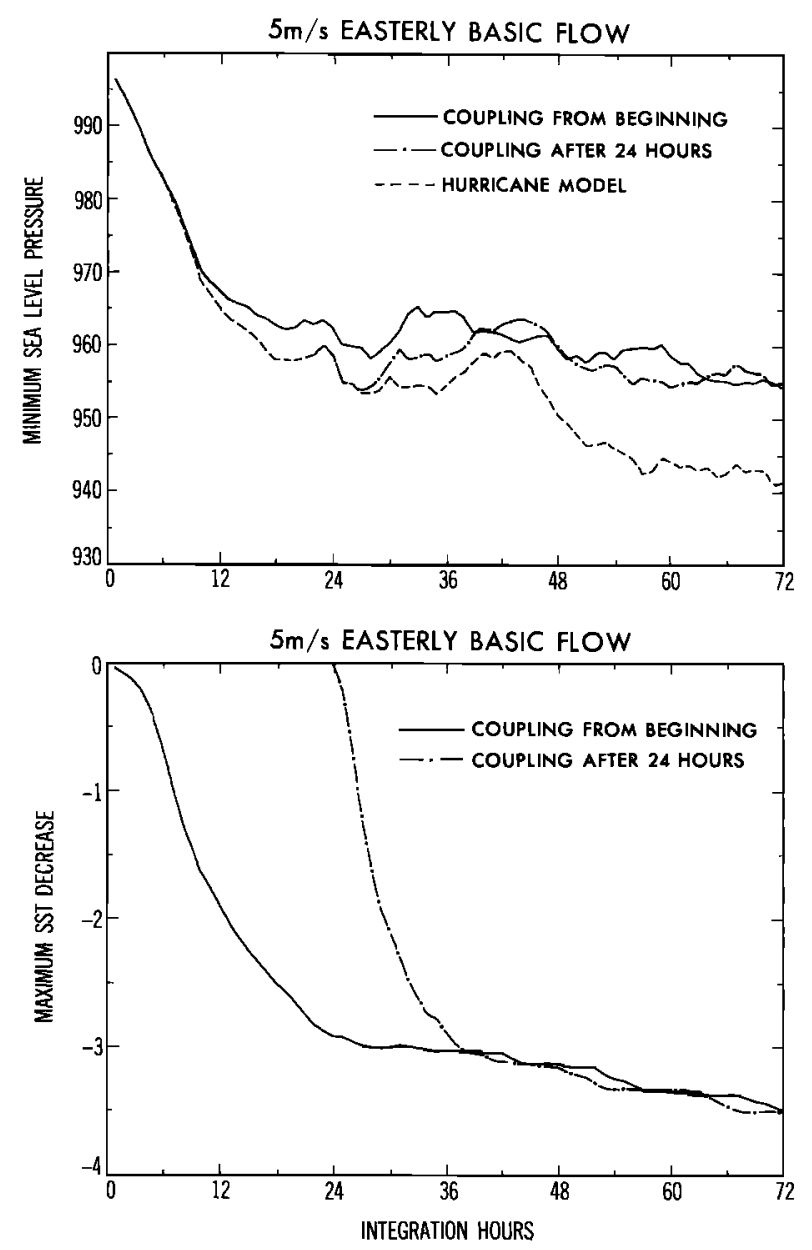

Fig. 14. Time series of minimum sea level pressure (top, hPa) for the $5 \mathrm{~m} \mathrm{~s}^{-1}$ easterly flow experiments run with hurricane-ocean coupling from the beginning (solid curve), without coupling (dashed curve), and with the coupling beginning at 24 hours (dashed-dotted curve). The maximum SST cooling $\left({ }^{\circ} \mathrm{C}\right.$ ) is also plotted (bottom) for the experiments with coupling from the beginning (solid line) and with the hurricane-ocean coupling beginning at 24 hours (dasheddotted curve).
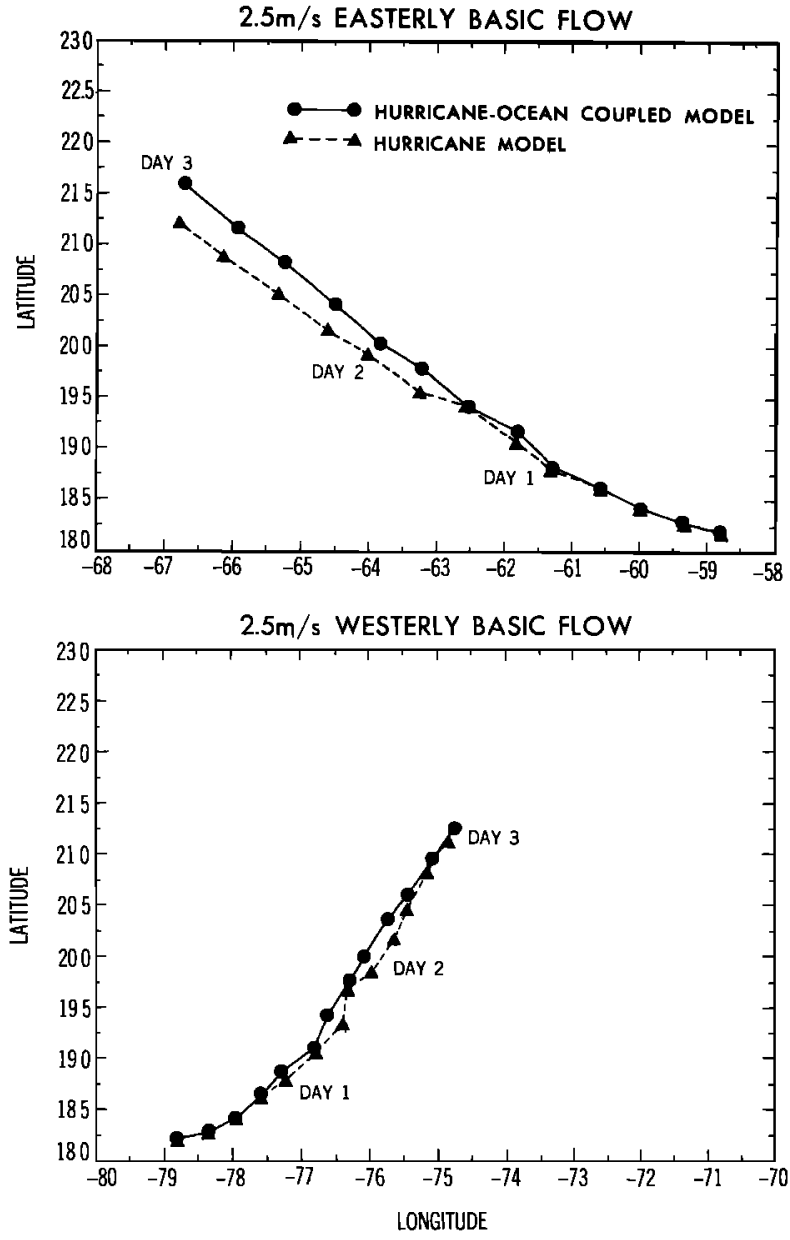

Fig. 15. The 72-hour storm tracks for the experiments with the $2.5 \mathrm{~m} \mathrm{~s}^{-1}$ easterly basic flow (top) and the $2.5 \mathrm{~m} \mathrm{~s}^{-1}$ westerly flow (bottom) run with (solid curve) and without (dashed curve) hurricane-ocean coupling. The storm positions at 6-hour intervals are indicated by circles (with coupling) or by triangles (without coupling).

basic flow experiments is that the slower-moving storms produced a progressively larger SST response and greater decrease of the total heat flux and hence a greater reduction in the tropical cyclone strength measured by both the minimum sea level pressure and the maximum low-level winds. These results were summarized in Table 3 for all of the experiments in this study.

The storm with the $5 \mathrm{~m} \mathrm{~s}^{-1}$ easterly basic flow experiment moved at about $6.1 \mathrm{~m} \mathrm{~s}^{-1}$ in a west-northwest direction, which is a typical translational speed for tropical cyclones in the deep tropics. Hence the maximum $3.2^{\circ} \mathrm{C}$ cooling produced in this experiment, which was confined to a relatively narrow area (about $2 / 3^{\circ}$ wide) just to the right of the storm track, may be considered a reasonable value for typical real-data cases and agreed well with observations. However, the actual ocean response is very sensitive to the specific vertical structure of the ocean, including the mixed layer depth. Therefore the magnitude of the ocean response can exhibit considerable variation from one location to another.

In a supplemental experiment the interaction with the ocean was introduced after integrating the tropical cyclone model with constant SST for 24 hours. By that time the tropical cyclone had reached the mature stage of develop- 

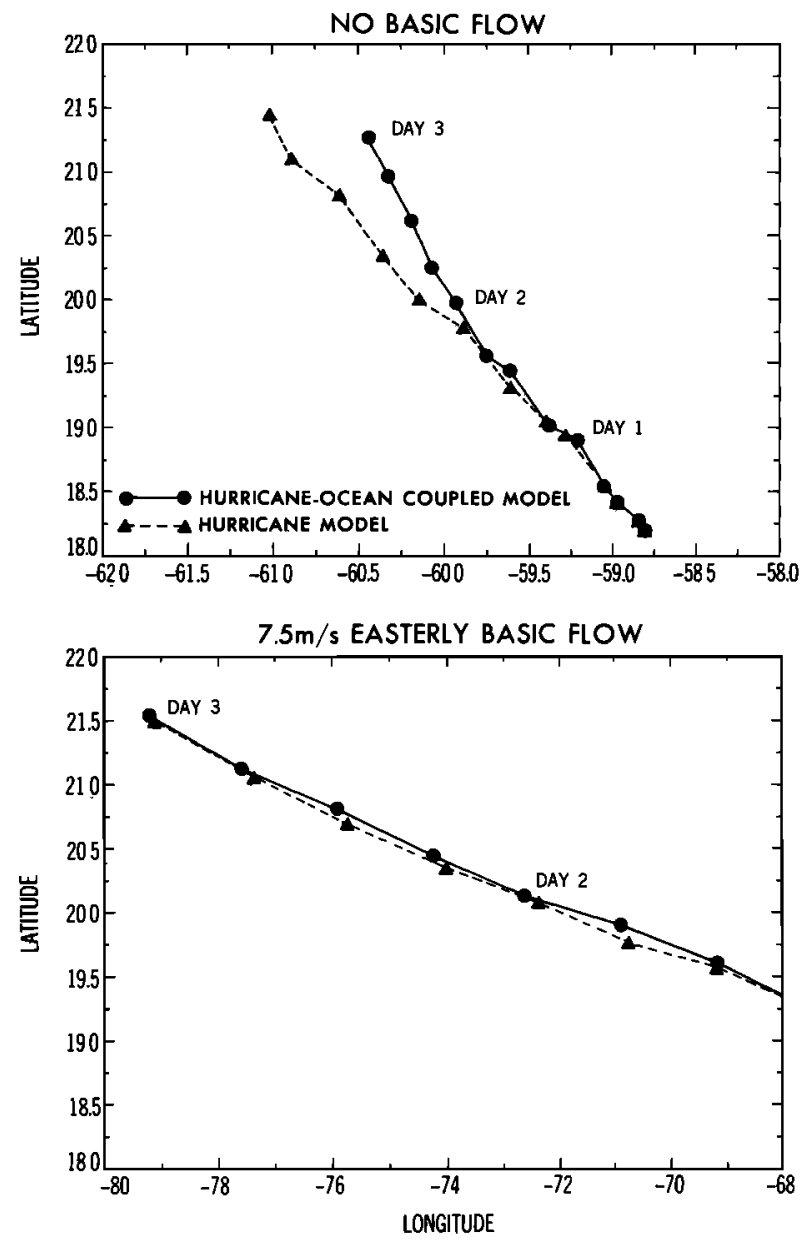

Fig. 16. The 72-hour storm tracks for the experiments with no basic flow (top) and with the $7.5 \mathrm{~m} \mathrm{~s}^{-1}$ easterly flow (bottom) run with (solid curve) and without (dashed curve) hurricane-ocean coupling. The storm positions at 6-hour intervals are indicated by circles (with coupling) or by triangles (without coupling).

ment with a minimum sea level pressure of $955 \mathrm{hPa}$. The purpose of this experiment was to determine the response time of a mature tropical cyclone to the effect of the ocean coupling. It was found that in contrast to previous numerical studies with coarser resolutions and simpler experimental designs the tropical cyclone immediately began to respond to the effect of the ocean coupling reaching the same intensity as the original coupled experiment within 12 hours. The final intensity and equilibrium state of the tropical cyclone for this case was found to be independent of the intensity of the storm at the time the ocean interaction began. This result will be useful in discussing the issue of initialization of ocean conditions.

It was also shown that the hurricane-ocean coupling significantly affected the track only for two of the cases with slowly moving storms. For the $2.5 \mathrm{~m} \mathrm{~s}^{-1}$ easterly basic flow the storm turned slightly more to the north after 36 hours and was located about $45 \mathrm{~km}$ to the north of the control case by 72 hours. The impact on the storm track was largest for the no basic flow case as the storm turned gradually more to the north and to the east by the end of the second day and was finally located over $70 \mathrm{~km}$ to the east-southeast of the control case by 72 hours. A possible explanation for this track deviation was the altering of the beta gyre related to a systematic weakening of the tangential flow at all radii of the storm induced by the interaction with the ocean. Only a small percentage of observed storms move with the slow propagation speed simulated in the no basic flow case. However, situations in which the steering flow is weak represent some of the most difficult cases in real track prediction. In these situations this effect may prove important to the accurate prediction of the storm track.

Although the experiments in this study were performed for an idealized experimental design and environment, they reveal some of the important effects that the ocean interaction may have on the behavior of actual tropical cyclones. In real cases the sensitivity shown of the storm to the sea surface cooling can be modulated by many other environmental factors such as the large-scale flow and vertical profiles of temperature or humidity. Some of these environmental factors may prohibit tropical cyclones from reaching their maximum potential intensity [e.g., Emanuel, 1988]. Tuleya and Kurihara [1982] showed numerically that these types of environmental conditions can also significantly alter the sensitivity of tropical storm genesis to the SST. It is even possible in some cases that the storm intensity will not significantly change despite large changes in the SST. However, the numerical experiments presented here indicate that the supply of latent energy to the storm will be significantly reduced in all cases in which cooling of the sea surface occurs. The results presented here also demonstrate that the SST decrease caused by the tropical cyclone-ocean coupling introduces an additional important mechanism that can reduce the maximum intensity of real tropical cyclones.

As the numerical models continue to improve, the effect of ocean interaction will probably become an important factor to consider for the successful forecast of tropical cyclone behavior. Thus a better understanding and evaluation of this effect is desirable. Future plans include expansion of the study of the effect of ocean interaction to some actual observed cases. The present numerical experiments were designed to simulate the effect of tropical cyclone-ocean interaction in the deep ocean. To study real data cases, the adaptation of a primitive equation model that includes the effect of bottom topography as well as detailed coastal

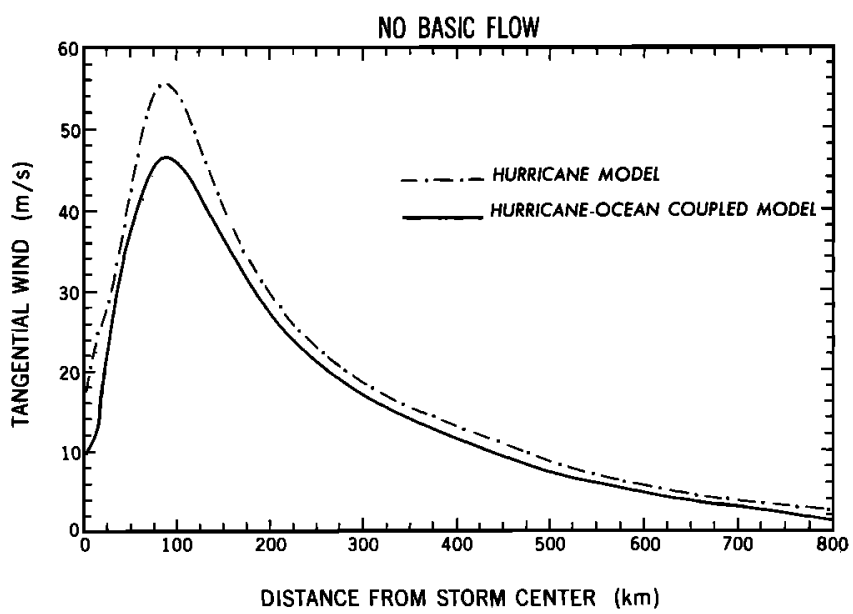

Fig. 17. The distribution of the circular averaged tangential wind at 72 hours for model level $14(\sigma=0.85)$ for the no basic flow experiments run with hurricane-ocean coupling (solid curve) and without coupling (dashed-dotted curve). 
geography is presently being considered. Analysis of these observed cases will be necessary in order to clarify the role of the tropical cyclone-ocean interaction in the real atmosphere and ocean.

\section{APPENDIX}

The ocean model used is formulated with the Boussinesq and hydrostatic approximations. The coordinate origin is located at the undisturbed sea surface with the vertical coordinate directed downward. The model is simplified by excluding the effect of horizontal diffusion and assuming constant salinity. A layered model is subject to kinematic and dynamic conditions at the boundaries and the layer interfaces. In the present model the turbulent momentum and the heat fluxes at the free surface are equal to the surface wind stress and total (latent and sensible) heat flux into the atmosphere, and pressure is equal to sea level atmospheric pressure. At the interface, between the mixed layer and the thermocline, the exchanges of mass, heat, and momentum take place through turbulent mixing which produces the entrainment. An important aspect of this exchange process is the energetics of the mixed layer and the radiation of turbulent energy into the thermocline. It is indicated from some theoretical and laboratory analyses (a recent review is given by Fernando [1991]) that the bulk of the energy fed into the mixed layer is trapped by the interfacial layer beneath the mixed layer. This energy is dissipated by wave breaking, and only an insignificant part of the energy is radiated to the thermocline. Accordingly, in the present model we assume that the turbulence caused by hurricane forcing is confined within the mixed layer above the discontinuity surface which is regarded as an infinitely thin interfacial layer. The turbulence in the mixed layer induces entrainment of mass, momentum, and heat across the bottom of the mixed layer. Consequently, the temperature change immediately below the mixed layer may result from entrainment. Since heat does not penetrate farther down in the model, the temperature of the layer interfaces within the thermocline and deep water remain unchanged. The potential role of bottom friction on the hurricane-generated currents is not considered in the present study. Accordingly, we limit our interest to the regions of the outer continental shelf and deep ocean.

The prognostic variables are the layer thicknesses $h_{i}$, horizontal current velocity in each layer $\mathbf{v}_{i},(i=1, \mathrm{~N}$ where $N$ is the number of model layers), mixed layer temperature (sea surface temperature) $T_{1}$, and temperature at the top of the thermocline $T_{2}$. Their time changes are governed by the equations of continuity, momentum, and heat balance, respectively, with the kinematic and dynamic conditions taken into account. In addition, the equation of state is used to relate the density to temperature.

The equations for the layer thicknesses are

$$
\begin{gathered}
h_{1 t}+\nabla \cdot\left(h_{1} \mathbf{v}_{1}\right)=w_{e} \\
h_{2 t}+\nabla \cdot\left(h_{2} \mathbf{v}_{2}\right)=-w_{e} \\
h_{i t}+\nabla \cdot\left(h_{i} \mathbf{v}_{i}\right)=0 \quad(i=3, N)
\end{gathered}
$$

where the subscript $t$ means time derivative and $w_{e}$ is the rate of entrainment into the mixed layer.

The momentum equations are written as

$$
\begin{aligned}
\left(h_{1} \mathbf{v}_{1}\right)_{t}+\nabla \cdot\left(\mathbf{v}_{1} h_{1} \mathbf{v}_{1}\right)+(f+ & \left.\frac{u_{1} \tan \theta}{a}\right) \mathbf{k} \times h_{1} \mathbf{v}_{1} \\
& =-\frac{1}{\rho_{0}} \mathbf{P}_{1}+\tau_{s}+w_{e} \mathbf{v}_{2},
\end{aligned}
$$

$$
\begin{array}{r}
\left(h_{2} \mathbf{v}_{2}\right)_{t}+\nabla \cdot\left(\mathbf{v}_{2} h_{2} \mathbf{v}_{2}\right)+\left(f+\frac{u_{2} \tan \theta}{a}\right) \mathbf{k} \times h_{2} \mathbf{v}_{2} \\
=-\frac{1}{\rho_{0}} \mathbf{P}_{2}-w_{e} \mathbf{v}_{2},
\end{array}
$$

$\left(h_{i} \mathbf{v}_{i}\right)_{t}+\nabla \cdot\left(\mathbf{v}_{i} h_{i} \mathbf{v}_{i}\right)$

$$
\begin{array}{r}
+\left(f+\frac{u_{i} \tan \theta}{a}\right) \mathbf{k} \times h_{i} \mathbf{v}_{i}=-\frac{1}{\rho_{0}} \mathbf{P}_{i} \\
(i=3, N)
\end{array}
$$

where $\tau_{s}$ is the wind stress vector, $a$ is the radius of the Earth, $\theta$ is the latitude, $\rho_{0}$ is the mean value of density, $\mathbf{P}_{i}$ is the gradient of pressure integrated over the $i$ layer and defined as

$$
\begin{gathered}
\mathbf{P}_{1}=g h_{1}\left[\rho_{1} \nabla \xi_{s}+\nabla p_{a}+\frac{1}{2} h_{1} \nabla \rho_{1}\right] \\
\mathbf{P}_{i}=g h_{i}\left[\rho_{i}^{\prime} \nabla \xi_{s}+\nabla p_{a}+\left(\rho_{i}^{\prime}-\rho_{1}\right) \nabla h_{1}+\sum_{j=2}^{i-1}\left(\rho_{i}^{\prime}-\rho_{j}^{\prime}\right) \nabla h_{j}\right. \\
\left.+\frac{1}{6}\left(\rho_{i+1}^{\prime}-\rho_{i}^{\prime}\right) \nabla h_{i}+h_{1} \nabla \rho_{1}+\frac{1}{2} h_{2} \nabla \rho_{2}\right] \\
(i=2, N)
\end{gathered}
$$

where $\rho_{i}^{\prime}=1 / 2\left(\rho_{i+1}+\rho_{i}\right), g$ is the acceleration of gravity, $p_{a}$ is the sea level atmospheric pressure. The sea surface displacement $\xi_{s}$ is determined by

$$
\xi_{s}=H-\sum_{i=1}^{N} h_{i}
$$

where $H$ is the mean ocean depth.

The model dynamics involves fast-propagating barotropic gravity waves. In the present study, for computational efficiency and also since the response of the deep ocean to hurricane forcing is primarily baroclinic [Geisler, 1970], we remove the above fast mode by using a reduced gravity approximation. Namely, we assume that the lowest model layer is at rest and infinitely deep. This requires vanishing of the pressure gradient for $h_{N} \rightarrow \infty$ in the relation (8). The resulting equation to determine the gradient of the sea surface elevation is given by

$$
\begin{array}{r}
\nabla \xi_{s}=-\frac{1}{\rho_{N}}\left[\nabla p_{a}+\left(\rho_{N}^{\prime}-\rho_{1}\right) \nabla h_{1}+\sum_{j=2}^{N-1}\left(\rho_{N}^{\prime}-\rho_{j}^{\prime}\right) \nabla h_{j}\right. \\
\left.+h_{1} \nabla \rho_{1}+\frac{1}{2} h_{2} \nabla \rho_{2}\right] .
\end{array}
$$

The equations for temperature at the mixed layer and at the top of the thermocline may be conveniently written as 


$$
\begin{gathered}
\left(h_{1} \Theta_{1}\right)_{t}+\nabla \cdot\left(\mathbf{v}_{1} h_{1} \Theta_{1}\right)=B_{s}+w_{e} \Theta_{2}, \\
\left(h_{2} \Theta_{2}\right)_{t}+\nabla \cdot\left(\mathbf{v}_{2} h_{2} \Theta_{2}\right)=-2 w_{e} \Theta_{2},
\end{gathered}
$$

where $\Theta_{1}=T_{1}-T_{3}, \Theta_{2}=T_{2}-T_{3}, T_{3}$ is the unchanged temperature at the base of the second layer; $B_{s}$ is the net heat flux at the sea surface divided by $\rho c_{p}$.

To close the system of equations, we determine the rate of entrainment $w_{e}$ with the use of a scheme developed by Deardorff [1983]. In his scheme, three mechanisms of turbulent kinetic energy production in the mixed layer are taken into consideration, i.e., production by near-surface winddriven shear, by velocity shear at the base of the mixed layer, and by convection due to the surface buoyancy fluxes. Scale velocities characterizing these mechanisms are $u_{*}=$ $\sqrt{\tau_{s} / \rho}$ (friction velocity), $\delta \mathrm{v}=\mathbf{v}_{1}-\mathbf{v}_{2}$ (velocity jump across the mixed layer base), and $w_{*}=\left(-\alpha g h_{1} B_{s}\right)^{1 / 3}$ (freeconvection velocity associated with the buoyancy flux; $\alpha$ is the thermal expansion coefficient), respectively. In addition, the velocity scale related to the temperature jump $\left(\delta T=T_{1}\right.$ $\left.-T_{2}\right)$ is expressed by $c_{i}=\left(\alpha g h_{1} \delta T\right)^{1 / 2}$. Then the ratio $w_{e}$ against $u_{*}$ (or $\delta \mathrm{v}, w_{*}$ ) can be expressed in terms of nondimensional parameters $R i_{\tau}=c_{i}^{2} / u_{*}^{2}, R i_{v}=c_{i}^{2} /(\delta \mathrm{v})^{2}$, and $R i_{*}=c_{i}^{2} / w_{*}^{2}$. Specifically, the following formula is derived from Deardorff's parameterization:

$$
\begin{aligned}
& \left(\frac{w_{e}}{u_{*}}\right)\left(\frac{R i_{q}}{R i_{\tau}}\right)^{1 / 2}=\left\{\left[0.1-0.06 \exp \left(-4 R i_{q}\right)\right]\right. \\
& \left.\cdot\left[R i_{q} / F_{h}\right]^{3 / 2}\left[\left(R i_{*}\right)^{-3 / 2}+F_{\eta} R i_{\tau}^{-3 / 2}\right]-0.14\right\} /\left\{1.2+0.3 R i_{q}\right. \\
& \left.\quad-0.3\left[1-0.93 \exp \left(-0.35 R i_{q}^{3 / 2}\right)\right] R i_{q} R i_{v}^{-1} F_{h}^{-1}\right\} \quad \text { (13) }
\end{aligned}
$$

where $F_{\eta}=1.8\left[1-2^{-1 / 2} f\left|\mathbf{v}_{1}\right| h_{1} u_{*}^{-2}\right]$ and $F_{h}=(1+$ $\left.1.9 R i_{q}\right)^{-0.4} ; R i_{q}$ is a nondimensional parameter based on the velocity scale involving the turbulent kinetic energy. Following Deardorff, we combine (13) with an empirically obtained formula

$$
\begin{aligned}
& \left(\frac{w_{e}}{u_{*}}\right)\left(\frac{R i_{q}}{R i_{\tau}}\right)^{1 / 2} \\
& = \begin{cases}0.66-0.52 R i_{q} & \text { if } R i_{q} \leq 0.52 \\
0.28 R i_{q}^{-1.34}-\exp \left(-5\left(R i_{q}-0.52\right)\right)\end{cases}
\end{aligned}
$$

and solve these equations for $w_{e}$ and $R i_{q}$, using the iterative method described in the appendix of Deardorff [1983].

The model equations are time integrated numerically on a staggered Arakawa B grid in an open ocean domain. The normal component of the current velocity is set to zero at the domain boundary. To reduce the influence of the boundaries on the solution, the domain size is chosen much larger than the area of hurricane forcing. In addition, we apply a Newtonian damping method to the six-grid-point zone near the boundaries [Kurihara and Bender, 1980]. The time integration proceeds with a splitting method developed by Marchuk [1982], the absolutely stable Crank-Nicolson scheme is used for the advection terms, and the scheme of "natural filter" for the adaptation terms. Ginis and Sutyrin [1993] show how the splitting method can be effectively applied for solving the nonlinear layered model equations.
Acknowledgments. The authors express their thanks to Jerry Mahlman for his continuous support of the Hurricane Dynamics Project at GFDL. They are also grateful to Tal Ezer and Robert E. Tuleya for their useful comments and criticisms of the original version of this manuscript and to Kerry Emanuel for his valuable suggestions. Finally, special thanks and credit is given to $P$. Tunison, K. Raphael, and J. Varanyak for helping in the preparation of the figures.

\section{REFERENCES}

Bender, M. A., R. E. Tuleya, and Y. Kurihara, A numerical study of the effect of a mountain range on a landfalling tropical cyclone, Mon. Weather Rev., 113, 567-582, 1985.

Bender, M. A., R. E. Tuleya, and Y. Kurihara, A numerical study of the effect of island terrain on tropical cyclones, Mon. Weather Rev., 115, 130-155, 1987.

Bender, M. A., R. J. Ross, R. E. Tuleya, and Y. Kurihara, Improvements in tropical cyclone track and intensity forecasts using the GFDL initialization system, Mon. Weather Rev., 121, 2046-2061, 1993.

Black, P. G., Ocean temperature change induced by tropical cyclones, Ph.D. dissertation, 278 pp., Pennsylvania State University, University Park, 1983.

Chang, S. W., The response of an axisymmetric model tropical cyclone to local variations of sea surface temperature, Mon. Weather Rev., 107, 662-666, 1979.

Chang, S. W., and R. A. Anthes, Numerical simulations of the ocean's nonlinear baroclinic response to translating hurricanes, $J$. Phys. Oceanogr., 8, 468-480, 1978.

Chang, S. W., and R. A. Anthes, The mutual response of the tropical cyclone and the ocean, J. Phys. Oceanogr., 9, 128-135, 1979.

Church, J. A., T. M. Joyce, and J. F. Price, Current and density observations across the wake of Hurricane Gay, J. Phys. Oceanogr., 19, 259-265, 1989.

Cooper, C., and J. D. Thompson, Hurricane-generated currents on the outer continental shelf, 1, Model formulation and verification, J. Geophys. Res., 94, 12,513-12,539, 1989.

Cornillon, P., L. Stramma, and J. F. Price, Satellite measurements of sea surface cooling during hurricane Gloria, Nature, 326, $373-375,1987$.

Deardorff, J. W., A multi-limit mixed layer entrainment formulation, J. Phys. Oceanogr., 13, 988-1002, 1983.

Emanuel, K. A., An air-sea interaction theory for tropical cyclones, 1, Steady-state maintenance, J. Atmos. Sci., 43, 585-604, 1986.

Emanuel, K. A., The maximum intensity of hurricanes, J. Atmos. Sci., 45, 1143-1155, 1988.

Fernando, H. J. S., Turbulent mixing in stratified fluids, Annu. Rev. Fluid Mech., 23, 455-493, 1991.

Fiorino, M., and R. L. Elsberry, Some aspects of vortex structure related to tropical cyclone motion, J. Atmos. Sci., 46, 975-990, 1989.

Geisler, J. E., Linear theory of the response of a two layer ocean to a moving hurricane, Geophys. Fluid Dyn., 1, 249-272, 1970.

Ginis, I., and Kh. Zh. Dikinov, Modeling of the Typhoon Virginia (1978) forcing on the ocean, Sov. Meteorol. and Hydrol. Engl. Transl., 7, 53-60, 1989.

Ginis, I., and G. G. Sutyrin, The hurricane-generated depthaveraged currents and sea surface effects, J. Phys. Oceanogr., in press, 1993.

Ginis, I., Kh. Zh. Dikinov, and A. P. Khain, A three dimensional model of the atmosphere and the ocean in the zone of a typhoon, Dokl. Akad. Nauk SSSR, 307, 333-337, 1989.

Johnson, A., and G. A. Speer, Data report: Buoy observations during Hurricane Belle, August 1976, 22 pp., Natl. Space Technol. Lab., NSTL Station, Miss., 1978.

Johnson, A., and G. W. Withee, Ocean data buoy measurements of Hurricane Eloise, Mar. Technol. Soc. J., 12, 14-20, 1978.

Khain, A. P., Three-dimensional numerical model of a tropical cyclone including $\beta$-effect, Atmos. Ocean. Phys., 24, 359-366, 1988.

Khain, A. P., and I. Ginis, The mutual response of a moving tropical cyclone and the ocean, Beitr. Phys. Atmos., 64, 125-141, 1991.

Kurihara, Y., A scheme of moist convective adjustment, Mon. Weather Rev., 101, 547-553, 1973. 
Kurihara, Y., and M. A. Bender, Use of a movable nested mesh model for tracking a small vortex, Mon. Weather Rev., 108, 1792-1809, 1980.

Kurihara, Y., and M. A. Bender, Structure and analysis of the eye of a numerically simulated tropical cyclone, J. Meteorol. Soc. Jpn., 60, 381-395, 1982.

Kurihara, Y., M. A. Bender, and R. J. Ross, An initialization scheme of hurricane models by vortex specification, Mon. Weather Rev., 121, 2030-2045, 1993.

Kurihara, Y., M. A. Bender, R. E. Tuleya, and R. J. Ross, Prediction experiments of Hurricane Gloria, 1985, using a multiply nested movable mesh model, Mon. Weather Rev., 118, 2185-2198, 1990.

Leipper, D. F., Observed ocean conditions and Hurricane Hilda, 1964, J. Atmos. Sci., 24, 182-196, 1967.

Marchuk, G. I., Methods of Numerical Mathematics, 510 pp., Springer-Verlag, New York, 1982.

Mellor, G. L., and T. Yamada, A hierarchy of turbulence closure models for planetary boundary layers, J. Atmos. Sci., 31, 17911806, 1974 .

Ogata, T., On the heat exchange between sea surface and lower atmosphere, J. Meteorol. Res., 12, 92-106, 1960.

Price, J. F., Upper ocean response to a hurricane, J. Phys. Oceanogr., 11, 153-175, 1981 .

Price, J. F., Inertial wave wake of a moving storm, 1, Scales, Energy budget and observations, J. Phys. Oceanogr., 13, 949965,1983

Pudov, V. D., Mesostructure of the temperature and current velocity fields of a baroclinic ocean layer in the wake of Typhoon Virginia, Oceanology, Engl. Transl., 20, 8-13, 1980.

Pudov, V. D., A. A. Varfolomeyev, and K. N. Fedorov, Vertical structure of the wake of a typhoon in the upper ocean, Oceanology, Engl. Transl., 18, 142-146, 1978.

Ramage, C. S., The typhoons of October 1970 in the South China Sea: Intensification, decay and ocean interaction, J. Appl. Meteorol., 13, 739-751, 1974

Sanford, T. B., P. G. Black, J. R. Haustein, J. W. Feeney, G. Z. Forristall, and J. F. Price, Ocean response to a hurricane, 1, Observations, J. Phys. Oceanogr., 17, 2065-2083, 1987.

Schramm, W. G., Airborne expendable bathythermograph observa- tions immediately before and after passage of Typhoon Phyllis in August of 1975, Tech. Rep. 79-95, 42 pp., Dep. of Navy, Monterey, Calif., 1979.

Shay, L. K., P. G. Black, J. D. Hawkins, R. L. Elsberry, and A. J. Mariano, Sea surface temperature response to Hurricane Gilbert, in Proceedings of the 19th Conference on Hurricanes and Tropical Meteorology, pp. 574-578, American Meteorological Society, Boston, Mass., 1991.

Stevenson, R. E., and R. S. Armstrong, Heat loss from the waters of the Northwest Gulf of Mexico during Hurricane Carla, Geofis. Int., 5, 49-55, 1965.

Stramma, L., P. Comillion, and J. F. Price, Satellite observations of sea surface cooling by hurricanes, J. Geophys. Res., 91, 5031$5035,1986$.

Sutyrin, G. G., and A. P. Khain, Interaction of the ocean and the atmosphere in the area of moving tropical cyclone, Dokl. Akad. Nauk SSSR, 249, 467-470, 1979.

Sutyrin, G. G., and A. P. Khain, On the effect of air-ocean interaction on intensity of moving tropical cyclone, Atmos. Oceanic Phys., 20, 697-703, 1984.

Tuleya, R. E., and Y. Kurihara, A note on the sea surface temperature sensitivity of a numerical model of tropical storm genesis, Mon. Weather Rev., 110, 2063-2069, 1982.

Tuleya, R. E., M. A. Bender, and Y. Kurihara, A simulation study of the landfall of tropical cyclones using a movable nested-grid model, Mon. Weather Rev., 112, 124-136, 1984.

Wright, R., Temperature structure across the Kuroshio before and after Typhoon Shirley, Tellus, 21, 409-413, 1969.

Wu, J., Wind-stress coefficients over sea surface from breeze to hurricane, J. Geophys. Res., 87, 9704-9706, 1982.

M. A. Bender and Y. Kurihara, Geophysical Fluid Dynamics Laboratory/NOAA, Princeton University, Princeton, NJ 08542.

I. Ginnis, Graduate School of Oceanography, University of Rhode Island, Narragansett, RI 02882.

(Received May 24, 1993;

revised August 14, 1993;

accepted August 17, 1993.) 